\title{
GENERIC SUBMANIFOLDS OF SASAKIAN MANIFOLDS
}

\author{
By Kentaro Yano and Masahiro Kon
}

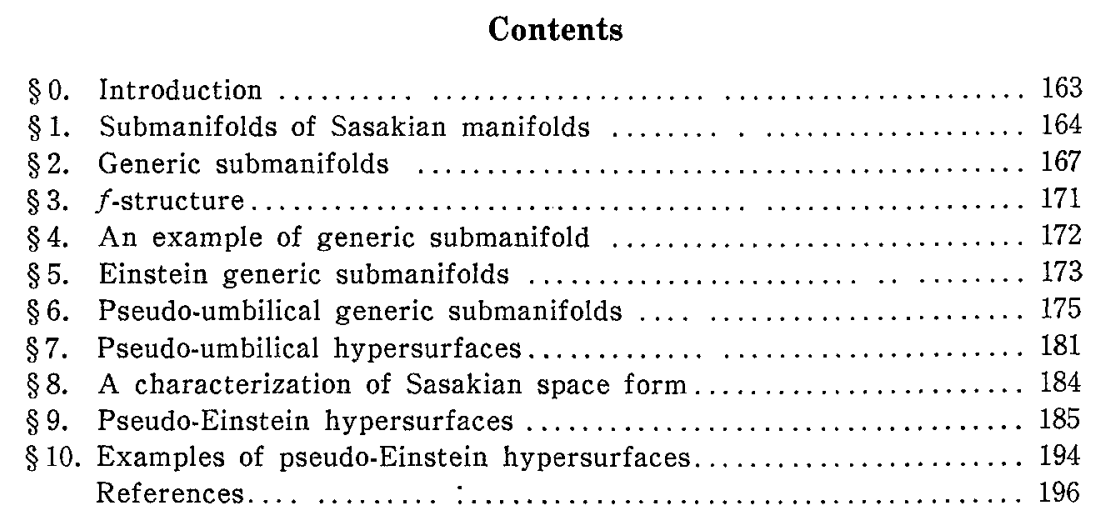

\section{$\S 0$. Introduction.}

In a previous paper [13], the present authors studied generic submanifolds immersed in complex space forms by the method of Riemannian fibre bundles.

The purpose of the present paper is to study generic submanifolds of Sasakian manifolds, especially those of Sasakian space forms.

In $\S 1$, we state some known results on submanifolds of Sasakian manifolds and study certain properties of the second fundamental forms of such submanifolds.

In $\S 2$, we define generic submanifolds of Sasakian manifolds and prove Propositions 2.1 and 2.2 on totally contact-umbilical generic submanifolds.

$\S 3$ is devoted to the study of the $f$-structure which a generic submanifold admits and to that of complete integrability of the distributions $\mathcal{L}$ and $\mathscr{T}$ associated with this $f$-structure.

In $\$ 4$, we construct an example of generic submanifold of a Sasakian space form and in $\S 5$ we prove Theorem 5.1 which characterizes complete generic minimal Einstein submanifolds of $S^{2 m+1}$ with parallel second fundamental form.

In $\S 6$, we define pseudo-umbilical submanifolds of Sasakian manifolds and prove propositions and theorems on pseudo-umbilical generic submanifolds and in $\S 7$ we study pseudo-umbilical hypersurfaces by the method of Riemannian fibre bundles.

Received November 21, 1978 
In $\$ 8$, we define what we call $P$-axiom and show that if a $(2 n+1)$-dimensional Sasakian manifold $M(n \geqq 2)$ satisfies the $P$-axiom, then $M$ is a Sasakian space form.

$\S 9$ is devoted to the study of what we call pseudo-Einstein hypersurfaces of $S^{2 n+1}$. We prove a series of lemmas and then Theorem 9.1 which says that a pseudo-Einstein hypersurface of $S^{2 n+1}(n \geqq 3)$ has two constant principal curvatures or four constant principal curvatures.

In the last $\S 10$ we give some examples of pseudo-Einstein hypersurfaces.

\section{$\S 1$. Submanifolds of Sasakian manifolds.}

Let $\vec{M}$ be a $(2 m+1)$-dimensional Sasakian manifold with structure tensors $(\phi, \xi, \eta, g)$. The structure tensors of $\bar{M}$ satisfy

$$
\begin{aligned}
& \phi^{2} X=-X+\eta(X) \xi, \quad \phi \xi=0, \quad \eta(\xi)=1, \quad \eta(\phi X)=0, \\
& g(\phi X, \phi Y)=g(X, Y)-\eta(X) \eta(Y), \quad \eta(X)=g(X, \xi)
\end{aligned}
$$

for any vector fields $X$ and $Y$ on $\vec{M}$. We denote by $\bar{\nabla}$ the operator of covariant differentiation with respect to the metric $g$ on $\bar{M}$. We then have

$$
\bar{\nabla}_{X} \xi=\phi X, \quad\left(\bar{\nabla}_{X} \phi\right) Y=\bar{R}(X, \xi) Y=-g(X, Y) \xi+\eta(Y) X
$$

for any vector fields $X$ and $Y$ on $\bar{M}, \bar{R}$ denoting the Riemannian curvature tensor of $\bar{M}$.

Let $M$ be an $(n+1)$-dimensional submanifold of $\bar{M}$. Throughout this paper, we assume that the submanifold $M$ is tangent to the structure vector field $\xi$ of $\bar{M}$.

We denote by the same $g$ the Riemannian metric tensor field induced on $M$ from that of $\bar{M}$. The operator of covariant differentiation with respect to the induced connection on $M$ will be denoted by $\nabla$. Then the Gauss and Weingarten formulas are given respectively by

$$
\bar{\nabla}_{X} Y=\nabla_{X} Y+B(X, Y) \text { and } \bar{\nabla}_{X} V=-A_{V} X+D_{X} V
$$

for any vector fields $X, Y$ tangent to $M$ and any vector field $V$ normal to $M$, where $D$ denotes the operator of covariant differentiation with respect to the linear connection induced in the normal bundle of $M$. $A$ and $B$ appearing here are both called the second fundamental forms of $M$ and are related by

$$
g(B(X, Y), V)=g\left(A_{V} X, Y\right) \text {. }
$$

A vector field $V$ normal to $M$ is said to be parallel if $D_{X} V=0$ for any vector field $X$ tangent to $M$. The mean curvature vector $\mu$ of $M$ is defined to be $\mu=(\operatorname{Tr} B) /(n+1), \operatorname{Tr} B$ denoting the trace of $B$. If $\mu=0$, then $M$ is said to be minimal. If the second fundamental form $B$ of $M$ is of the form $B(X, Y)=$ $g(X, Y) \mu$, then $M$ is said to be totally umbilical. In particular, if the second fundamental form $B$ vanishes identically, then $M$ is said to be totally geodesic. If the second fundamental form $B$ of $M$ is of the form 


$$
B(X, Y)=[g(X, Y)-\eta(X) \eta(Y)] \alpha+\eta(X) B(Y, \xi)+\eta(Y) B(X, \xi)
$$

for any vector fields $X$ and $Y$ tangent to $M, \alpha$ being a vector field normal to $M$, then $M$ is said to be totally contact-umbilical. The notion of totally contactumbilical submanifolds of Sasakian manifolds corresponds to that of totally umbilical submanifolds of Kaehlerian manifolds (see [3]). Moreover, if $\alpha=0$, that is, if $B$ is of the form

$$
B(X, Y)=\eta(X) B(Y, \xi)+\gamma_{(}(Y) B(X, \xi),
$$

then $M$ is said to be totally contact-geodeszc. The notion of totally contactgeodesic submanifolds of Sasakian manifolds corresponds to that of totally geodesic submanifolds of Kaehlerian manifolds.

Let $R$ be the Riemannian curvature tensor field of $M$. Then, for any vector fields $X, Y$ and $Z$ tangent to $M$, we have

$$
\bar{R}(X, Y) Z=R(X, Y) Z-A_{B(Y, Z)} X+A_{B(X, Z)} Y+\left(\nabla_{X} B\right)(Y, Z)-\left(\nabla_{Y} B\right)(X, Z),
$$

where the covariant derivative $\nabla_{X} B$ of $B$ is defined to be

$$
\left(\nabla_{X} B\right)(Y, Z)=D_{X}(B(Y, Z))-B\left(\nabla_{X} Y, Z\right)-B\left(Y, \nabla_{X} Z\right)
$$

for any vector fields $X, Y$ and $Z$ tangent to $M$. If $\nabla_{X} B=0$ for any vector field $X$ tangent to $M$, then the second fundamental form $B$ of $M$ is said to be parallel. From (1.3), we have equation of Gauss

$$
\begin{aligned}
g(\bar{R}(X, Y) Z, W)= & g(R(X, Y) Z, W)-g(B(X, W), B(Y, Z)) \\
& +g(B(Y, W), B(X, Z))
\end{aligned}
$$

for any vector fields $X, Y, Z$ and $W$ tangent to $M$. Taking the normal component of (1.3), we have equation of Codazzi

$$
(\bar{R}(X, Y) Z)^{2}=\left(\nabla_{X} B\right)(Y, Z)-\left(\nabla_{Y} B\right)(X, Z),
$$

$(\bar{R}(X, Y) Z)^{\perp}$ denoting the normal component of $\bar{R}(X, Y) Z$. We now define the curvature tensor $R^{\perp}$ of the normal bundle of $M$ by

$$
R^{\perp}(X, Y) V=D_{X} D_{Y} V-D_{Y} D_{X} V-D_{[X, Y]} V
$$

$X, Y$ being vector fields tangent to $M$ and $V$ a vector field normal to $M$. Then we have equation of Ricci

$$
g(\bar{R}(X, Y) U, V)=g\left(R^{1}(X, Y) U, V\right)+g\left(\left[A_{V}, A_{U}\right] X, Y\right),
$$

where $\left[A_{V}, A_{U}\right]=A_{V} A_{U}-A_{U} A_{V}$.

If $R^{\perp}=0$, then the normal connection of $M$ is said to be flat (or trivial).

For any vector field $X$ tangent to $M$, we put

$$
\phi X=P X+F X,
$$

where $P X$ is the tangential part and $F X$ the normal part of $\phi X$. Then $P$ is an endomorphism on the tangent bundle $T(M)$ and $F$ is a normal bundle valued 1 -form on the tangent bundle. 
If $\phi T_{x}(M) \subset T_{x}(M)$ at each point $x$ of $M$, then $M$ is called an invarnant submanifold of $\bar{M}$. Any invariant submanifold of a Sasakian manifold is a Sasakian manifold. If $M$ is invariant, then $F$ in (1.8) vanishes identically. If $\phi T_{x}(M) \subset T_{x}(M)^{-}$at each point $x$ of $M$, then $M$ is called an anti-invariant submanifold of $\bar{M}$. If $M$ is anti-invariant, then $P$ in (1.8) vanishes identically. have

If the ambient manifold $\bar{M}$ is of constant $\phi$-sectional curvature $k$, then we

$$
\begin{aligned}
\bar{R}(X, Y) Z= & \frac{1}{4}(k+3)[g(Y, Z) X-g(X, Z) Y]+\frac{1}{4}(k-1)[\eta(X) \eta(Z) Y \\
& -\eta(Y) \eta(Z) X+g(X, Z) \eta(Y) \xi-g(Y, Z) \eta(X) \xi+g(\phi Y, Z) \phi X \\
& -g(\phi X, Z) \phi Y-2 g(\phi X, Y) \phi Z]
\end{aligned}
$$

for any vector fields $X, Y$ and $Z$ on $\bar{M}$. In this case $\bar{M}$ is called a Sasakzan space form and is denoted by $\bar{M}^{2 m+1}(k)$.

Let $M$ be an $(n+1)$-dimensional submanifold of a Sasakian space form $\bar{M}^{2 m+1}(k)$. Then (1.3), (1.6), (1.7) and (1.9) imply

$$
\begin{aligned}
& R(X, Y) Z= \frac{1}{4}(k+3)[g(Y, Z) X-g(X, Z) Y]+\frac{1}{4}(k-1)\left[r_{Y}(X) \eta(Z) Y\right. \\
&-\eta(Y) \eta(Z) X+g(X, Z) \eta(Y) \xi-g(Y, Z) \eta(X) \xi+g(P Y, Z) P X \\
&\quad-g(P X, Z) P Y-2 g(P X, Y) P Z]+A_{B(Y, Z)} X-A_{B(X, Z)} Y \\
&\left(\nabla_{X} B\right)(Y, Z)-\left(\nabla_{Y} B\right)(X, Z) \\
&=\frac{1}{4}(k-1)[g(P Y, Z) F X-g(P X, Z) F Y-2 g(P X, Y) F Z], \\
& \frac{1}{4}(k-1)[g(F Y, U) g(F X, V)-g(F X, U) g(F Y, V)-2 g(P X, Y) g(\phi U, V)] \\
&= g\left(R^{\perp}(X, Y) U, V\right)+g\left(\left[A_{V}, A_{U}\right] X, Y\right) .
\end{aligned}
$$

In the following, we study certain properties of the second fundamental form $B$ of $M$. Since the structure vector field $\xi$ is tangent to $M$, for any vector field $X$ tangent to $M$, we have

from which

$$
\bar{\nabla}_{X} \xi=\phi X=\nabla_{X} \xi+B(X, \xi),
$$

$$
P X=\nabla_{X} \xi, \quad F X=B(X, \xi) .
$$

Especially, we have

$$
B(\xi, \xi)=0
$$

Proposition 1.1. Let $M$ be an $(n+1)$-dimensional submanifold of a $(2 m+1)$ dimensional Sasakian manifold $\bar{M}$. If $M$ is totally umbilical, then $M$ is a totally geodesic, invariant submanifold of $\bar{M}$. 
Proof. Since $M$ is totally umbilical, the second fundamental form $B$ of $M$ is of the form $B(X, Y)=g(X, Y) \mu$. From this and (1.14) we have $B(\xi, \xi)=$ $g(\xi, \xi) \mu=0$ and hence $\mu=0$. Thus $B(X, Y)=0$ and consequently $M$ is a totally geodesic submanifold of $\bar{M}$. Moreover, the second equation of (1.13) implies $F X=B(X, \xi)=0$. This shows that $M$ is an invariant submanifold of $\bar{M}$.

Let $X$ and $Y$ be vector fields tangent to $M$. From the Gauss and Weingarten formulas we have

$$
\phi B(X, Y)=\left(\nabla_{Y} P\right) X-A_{F X} Y+B(Y, P X)+\left(\nabla_{Y} F\right) X+g(X, Y) \xi-\eta(X) Y,
$$

where we have defined $\left(\nabla_{Y} P\right) X$ and $\left(\nabla_{Y} F\right) X$ respectively by

$$
\left(\nabla_{Y} P\right) X=\nabla_{Y}(P X)-P \nabla_{Y} X \text { and }\left(\nabla_{Y} F\right) X=D_{Y}(F X)-F \nabla_{Y} X \text {. }
$$

Since $B$ is symmetric, we have

$$
\begin{aligned}
& \left(\nabla_{Y} P\right) X+B(Y, P X)-A_{F X} Y+\left(\nabla_{Y} F\right) X-\eta(X) Y \\
& \quad=\left(\nabla_{X} P\right) Y+B(X, P Y)-A_{F Y} X+\left(\nabla_{X} F\right) Y-\eta(Y) X .
\end{aligned}
$$

Comparing the tangential and normal parts in the equation above, we obtain

$$
\left(\nabla_{X} P\right) Y-\left(\nabla_{Y} P\right) X=A_{F^{\prime} Y} X-A_{F X} Y+\eta(Y) X-\eta(X) Y,
$$

and

$$
\left(\nabla_{X} F\right) Y-\left(\nabla_{X} F\right) X=B(Y, P X)-B(X, P Y)
$$

respectively.

\section{$\S 2$. Generic submanifolds.}

A submanifold $M$ of a Sasakian manifold $\bar{M}$ is called a generic submanfold of $\bar{M}$ if $\phi T_{x}(M)^{\perp} \subset T_{x}(M)$ for all point $x$ of $M$ and if $\xi$ is tangent to $M$. Especially, if $\phi T_{x}(M)^{\perp}=T_{x}(M)-\{\xi\}$, then a generic submanifold $M$ is an antiinvariant submanifold such that $2 \operatorname{dim} M-1=\operatorname{dim} \bar{M}$. If $\operatorname{dim} T_{x}(M)^{\perp}=1$, that is, if $M$ is a hypersurface of $\bar{M}$, then $M$ is obviously a generic submanifold.

Let $M$ be a hypersurface of a Sasakian manifold $\bar{M}$. We denote by $C$ the unit normal of $M$ in $\bar{M}$. For any vector field $X$ tangent to $M$, we have

$$
\phi X=P X+u(X) C, \quad u(X) C=F X,
$$

where we have put

$$
\phi C=-U, \quad u(X)=g(U, X) .
$$

From (2.1) we find

$$
P^{2} X=-X+u(X) U+\eta(X) \xi
$$

Moreover, we have

$$
P U=0, \quad u(\xi)=0, \quad u(U)=1 .
$$

We denote the second fundamental form of $M$ by $A$ in place of $A_{C}$ to 
simplify the notation. Then the Gauss and Weingarten formulas are given respectively by

We also have

$$
\bar{\nabla}_{X} Y=\nabla_{X} Y+g(A X, Y) C \text { and } \bar{\nabla}_{X} C=-A X
$$

$$
\begin{gathered}
\nabla_{X} U=P A X, \quad A \xi=U, \\
\left(\nabla_{X} P\right) Y=\eta(Y) X+u(Y) A X-g(X, Y) \xi-g(A X, Y) U .
\end{gathered}
$$

Let $M$ be an $(n+1)$-dimensional generic submanifold of a $(2 m+1)$-dimensional Sasakian manifold $\bar{M}$. Then the tangent space $T_{x}(M)$ of $M$ is decomposed as follows :

$$
T_{x}(M)=H_{x}(M) \oplus \phi T_{x}(M)^{\perp}
$$

at each point $x$ of $M$, where $H_{x}(M)$ is the orthogonal complement of $\phi T_{x}(M)^{\perp}$ in $T_{x}(M)$. Thus we see that

Applying $\phi$ to $(1.8)$, we find

$$
\phi H_{x}(M)=H_{x}(M)-\{\xi\} .
$$

from which we have

$$
-X+\eta(X) \xi=P^{2} X+F P X+\phi F X
$$

$$
\begin{gathered}
F P X=0, \\
P^{2} X=-X+\eta(X) \xi-\phi F X .
\end{gathered}
$$

From (1.15) we have

$$
\begin{aligned}
\phi B(X, Y)= & \left(\nabla_{Y} P\right) X-A_{F X} Y+g(X, Y) \xi-\eta(X) Y, \\
& B(Y, P X)=-\left(\nabla_{Y} F\right) X .
\end{aligned}
$$

Let $V$ be a vector field normal to $M$. Then we find

$$
\begin{gathered}
\nabla_{X} \phi V=-P A_{Y} X+\phi D_{X} V, \\
B(X, \phi V)=-F A_{V} X .
\end{gathered}
$$

We notice that $P \phi T_{x}(M)^{\perp}=0$ and $\phi P T_{x}(M) \subset H_{x}(M)$. For any vector field $X$ tangent to $M$ and any $Y \in \phi T_{x}(M)^{\perp}$, we have

$$
\left(\nabla_{X} P\right) Y=\nabla_{X}(P Y)-P \nabla_{X} Y=-P \nabla_{X} Y \in H(M) .
$$

For any vector field $X$ tangent to $M$ and any $Y, Z \in \phi T(M)^{\perp}$, we have

$$
\begin{aligned}
g(\phi B(X, Y), Z) & =g\left(\left(\nabla_{X} P\right) Y, Z\right)-g\left(A_{F Y} X, Z\right)+g(X, Y) r(Z)-g(X, Z) \eta(Y) \\
& =-g\left(A_{F^{\prime} Y} Z, X\right) .
\end{aligned}
$$

On the other hand, we have

$$
g(\phi B(X, Y), Z)=-g(B(X, Y), F Z)=-g\left(A_{F Z} Y, X\right) .
$$

Therefore, we see that $g\left(A_{F Y} Z, X\right)=g\left(A_{F Z} Y, X\right)$, from which $A_{F Y} Z=A_{F Z} Y$ for any $Y, Z \in \phi T_{x}(M)^{\llcorner}$. Thus we have 
Lemma 2.1. Let $M$ be a genernc submanifold of a Sasakuan manfold $\bar{M}$. Then we have

$$
A_{F Y} Z=A_{F Z} Y
$$

for any rector fields $Y$ and $Z$ in $\phi T(M)^{\perp}$.

In the following we denote by $p$ the codimension of $M$, i.e., we put $p=2 m-n$.

Proposition 2.1. Let $M$ be a genernc submanfold of a Sasakzan manfold $\bar{M}$. If $p \geqq 2$ and $M$ is totally contact-umbilical, then $M$ is totally contact-geodesic.

Proof. First of all, using (2.13), we have

$$
g\left(A_{\alpha} X, X\right)=-g\left(A_{F x} \phi \alpha, X\right), \quad X \in \phi T_{x}(M)^{\perp},
$$

where $\alpha$ is the normal vector appearing in (1.1). From (1.1) we find

$$
B(X, X)=[g(X, X)-\eta(X) \eta(X)] \alpha+2 \eta(X) F X=g(X, X) \alpha
$$

for any $X \in \phi T_{x}(M)^{\perp}$. Thus we have

$$
\begin{aligned}
& g\left(A_{\alpha} X, X\right)=g(B(X, X), \alpha)=g(X, X) g(\alpha, \alpha), \\
& -g\left(A_{F Y} \phi \alpha, X\right)=-g(B(\phi \alpha, X), F X)=-g(\phi \alpha, X) g(\alpha, F X),
\end{aligned}
$$

from which

$$
g(X, X) g(\alpha, \alpha)=-g(\phi \alpha, X) g(\alpha, F X)=g(\alpha, F X) g(\alpha, F X)
$$

for $X \in \phi T_{x}(M)^{\perp}$. Since $p \geqq 2$, we can take $X$ such that $g(\alpha, F X)=0$. Thus we have $\alpha=0$ and hence $M$ is totally contact-geodesic.

Proposition. 2.2. Let $M$ be an $(n+1)$-dimensional $(n \geqq 3)$ generuc submamfold of a Sasakian space form $\bar{M}^{2 m+1}(k)$. If $M$ is totally contact-umbilical and if $n>m$, then $k=-3$.

Proof. If $p \geqq 2$, then Proposition 2.1 implies that $M$ is totally contact-geodesic. Thus the second fundamental form $B$ of $M$ is of the form

From this we find

$$
B(X, Y)=\eta(X) F Y+\eta(Y) F X \text {. }
$$

$$
\left(\nabla_{X} F\right) Y=-B(X, P Y)=-\eta(X) F P Y-\eta(P Y) F X=0 .
$$

Therefore we have

$$
\begin{aligned}
& \left(\nabla_{X} B\right)(Y, Z)=g(Y, P X) F Z+g(Z, P X) F Y \\
& \left(\nabla_{Y} B\right)(X, Z)=g(X, P Y) F Z+g(Z, P Y) F X
\end{aligned}
$$

From these equations and (1.11) we find 


$$
\begin{aligned}
& g(Z, P X) F Y-g(Z, P Y) F X+2 g(Y, P X) F Z \\
& \quad=\frac{1}{4}(k-1)[g(P Y, Z) F X-g(P X, Z) F Y-2 g(P X, Y) F Z],
\end{aligned}
$$

from which

$$
\frac{1}{4}(k+3)[g(P Y, Z) F X-g(P X, Z) F Y-2 g(P X, Y) F Z]=0 .
$$

Since $n>m, \operatorname{dim} H_{x}(M)>1$, we can take $Y$ such that $Y \in H_{x}(M)$ and put $Z=P Y$. Then $F Y=0$ and $F Z=F P Y=0$. Thus we have

$$
\frac{1}{4}(k+3) g(P Y, P Y) F X=0,
$$

which implies that $k=-3$.

In the following, we assume that $p=1$. Then we have

$$
F A X=B(X, U), \quad B(U, \xi)=F U=C .
$$

In this case, $(1.11)$ reduces to

$$
\begin{aligned}
& g\left(\left(\nabla_{X} A\right) Y, Z\right)-g\left(\left(\nabla_{Y} A\right) X, Z\right) \\
= & \frac{1}{4}(k-1)[g(P Y, Z) g(F X, C)-g(P X, Z) g(F Y, C)-2 g(P X, Y) g(F Z, C)] .
\end{aligned}
$$

Putting $Z=U$ in (2.15), we find

$$
g\left(\left(\nabla_{X} A\right) Y, U\right)-g\left(\left(\nabla_{Y} A\right) X, U\right)=-\frac{1}{2}(k-1) g(P X, Y),
$$

because of $g(P Y, U)=g(P X, U)=0$. On the other hand, we have

from which

$$
\left(\nabla_{X} A\right) U=\nabla_{X}(A U)-A P A X, \quad A U=g(C, \alpha) U+\xi,
$$

and hence

$$
\left(\nabla_{X} A\right) U=g\left(C, D_{X} \alpha\right) U+g(C, \alpha) P A X+P X-A P A X
$$

$$
\begin{aligned}
& g\left(\left(\nabla_{X} A\right) Y, U\right)-g\left(\left(\nabla_{Y} A\right) X, U\right)=g\left(Y,\left(\nabla_{X} A\right) U\right)-g\left(X,\left(\nabla_{Y} A\right) U\right) \\
& =g\left(C, D_{X} \alpha\right) g(U, Y)+g(C, \alpha) g(P A X, Y)+g(P X, Y)-g(A P A X, Y) \\
& \quad-g\left(C, D_{Y} \alpha\right) g(U, X)-g(C, \alpha) g(P A Y, X)-g(P Y, X)+g(A P A Y, X) .
\end{aligned}
$$

If we take here $X, Y$ such that $\eta(X)=\eta(Y)=0$ and $u(X)=u(Y)=0$, then we have

$$
\begin{aligned}
& g\left(\left(\nabla_{X} A\right) Y, U\right)-g\left(\left(\nabla_{Y} A\right) X, U\right) \\
& \quad=g(C, \alpha) g(P A X, Y)-g(A P A X, Y)-g(C, \alpha) g(P A Y, X) \\
& \quad+g(A P A Y, X)-2 g(P Y, X) .
\end{aligned}
$$

Moreover, we obtain the equations :

$$
g(A P A X, Y)=g(C, \alpha) g(P A X, Y), \quad g(A P A Y, X)=g(C, \alpha) g(P A Y, X) .
$$

From these equations we have 


$$
g\left(\left(\nabla_{X} A\right) Y, U\right)-g\left(\left(\nabla_{Y} A\right) X, U\right)=-2 g(P Y, X) .
$$

From this and (2.16) we have

$$
-\frac{1}{2}(k-1) g(P X, Y)=-2 g(P Y, X),
$$

from which

$$
\frac{1}{2}(k+3) g(P X, Y)=0 .
$$

Since $n \geqq 3$, we can put $Y=P X$ and so we obtain $k=-3$. Thus we have $k:=-3$ for any codimension.

\section{§3. $f$-structure}

Let $M$ be an $(n+1)$-dimensional generic submanifold of a $(2 m+1)$-dimensional Sasakian manifold $\bar{M}$. From (2.8) we have

$$
P^{3}+P=0 .
$$

On the other hand, we see that $\operatorname{rank} P=\operatorname{dim} M-\operatorname{codim} M-1=2(n-m)$ everywhere on $M$. Consequently, $P$ defines an $f$-structure of rank $2(n-m)$ (see [10]).

We now consider the distributions $\mathcal{L}$ and $\mathscr{I}$ respectively defined by

and

$$
\mathcal{L}_{x}=\left\{X \in T_{x}(M): F X=0\right\},
$$

$$
I_{x}=\left\{X \in T_{x}(M): P X=0 \text { and } \eta(X)=0\right\} .
$$

The distribution $\mathcal{L}$ is $(2 n-2 m+1)$-dimensional and the distribution $\mathscr{L}(2 m-n)$ dimensional. We study the integrability conditions of $\mathcal{L}$ and $\mathscr{I}$. First of all, we prove

Proposition 3.1. Let $M$ be an $(n+1)$-dimensional genernc submanifold of a $(2 m+1)$-dimensional Sasakian manifold $\bar{M}$. Then the distribution $\mathscr{I}$ is completely integrable and its maximal integral submantold $T$ is a $(2 m-n)$-dimenstonal ant $l_{-}$ invariant submanifold of $\bar{M}$ normal to $\xi$.

Proof. Let $X$ and $Y$ be vector fields in the distribution $\mathcal{I}$. Then (1.16) and (2.13) imply

Moreover we have

$$
\begin{aligned}
P[X, Y] & =P \nabla_{X} Y-P \nabla_{Y} X=\left(\nabla_{Y} P\right) X-\left(\nabla_{X} P\right) Y \\
& =A_{F X} Y-A_{F Y} X=0 .
\end{aligned}
$$

$$
\eta([X, Y])=-g(F X, Y)+g(F Y, X)=0 .
$$

Thus we see that $[X, Y] \in \mathscr{I}$ and hence $\mathscr{I}$ is completely integrable. We also see, from the construction, that the integral submanifold $T$ is anti-invariant with respect to $\phi$ and is normal to $\xi$. 
Proposition 3.2. Let $M$ be an $(n+1)$-dimensional generic submanifold of a $(2 m+1)$-dimensional Sasakian manfold $\bar{M}$. Then the distribution $\mathcal{L}$ is completely integrable if and only if

$$
B(P X, Y)=B(X, P Y)
$$

for any vector fields $X$ and $Y$ in $\mathcal{L}$.

Proof. Let $X$ and $Y$ be in $\mathcal{L}$. Then (1.17) implies that

$$
\begin{aligned}
F[X, Y] & =F \nabla_{X} Y-F \nabla_{Y} X=\left(\nabla_{Y} F\right) X-\left(\nabla_{X} F\right) Y \\
& =B(X, P Y)-B(Y, P X) .
\end{aligned}
$$

Therefore, $\mathcal{L}$ is completely integrable if and only if (3.2) holds.

If $\mathcal{L}$ is completely integrable, then its maximal integral submanifold $L$ is a $(2 n-2 m+1)$-dimensional invariant submanifold of $\vec{M}$.

\section{$\S 4$. An example of generic submanif old.}

In this section we give an example of generic submanifold of a Sasakian space form.

Let $C^{m+1}$ be a complex $(m+1)$-dimensional number space. We consider an odd-dimensional unit sphere $S^{2 m+1}$ in $C^{m+1}$. Then $S^{2 m+1}$ admits a Sasakian structure $(\phi, \xi, \eta, g)$ as follows. Let $v$ be the position vector representing a point of $S^{2 m+1}$ in $C^{m+1}$. Then the structure vector field of $S^{2 m+1}$ is given by $\xi=J v, J$ denoting the almost complex structure of $C^{m+1}$. Consider the orthogonal projection

$$
\pi: T_{x}\left(C^{m+1}\right) \rightarrow T_{x}\left(S^{2 m+1}\right),
$$

and put $\phi=\pi \cdot J$. We denote by $\eta$ the 1 -form dual to $\xi$ and by $g$ the standard metric tensor field on $S^{2 m+1}$. Then, for any vector field $X$ tangent to $S^{2 m+1}$, we have

$$
\phi X=J X+r_{\lambda}(X) v .
$$

We now consider the following immersion:

$$
S^{m_{1}}\left(\sqrt{\left.m_{1} / n\right)} \times \cdots \times S^{m_{k}}\left(\sqrt{ } m_{k} / n\right) \rightarrow S^{n+k-1}, \quad n=\sum_{i=1}^{k} m_{\imath} .\right.
$$

We assume that $m_{1}, \cdots, m_{k}$ are odd. Then $n+k-1$ is also odd. Let $v_{2}$ be a point of $S^{m_{i}}\left(\sqrt{m_{2} / n}\right)$ in $R^{m_{i}+1}=C^{\left(m_{i}+1\right) / 2} . \quad S^{m_{i}}\left(\sqrt{m_{2} / n}\right)$ is a real hypersurface of $C^{\left(m_{i}+1 / 2\right)}$ with unit normal $\sqrt{n / m_{\imath}} v_{\imath}$. Thus $v=\left(v_{1}, \cdots, v_{k}\right)$ is a unit vector in $R^{n+k}=C^{(n+k) i_{2}}$. This defines a minimal immersion of $M_{m_{1}, \ldots, m_{k}}=\Pi S^{m_{i}}\left(\sqrt{ } m_{\imath} / n\right)$ into $S^{n+k-1}$. We restrict the almost complex structure of $C^{(n+k) / 2}$ to $C^{\left(m_{i}+1\right) / 2}$. Then $J v_{2}$ is tangent to $S^{m_{i}}\left(\sqrt{m_{2}} / n\right)$. Thus $J v$ is tangent to $M_{m_{1}, \ldots, m_{k}}$. We then consider the normal space of $M_{m_{1}, \ldots, m_{k}}$ in $S^{n+k-1}$ which is the orthogonal complement of the 1-dimensional space $\langle v\rangle$ spanned by $v$ in the space $\left\langle v_{1}, \cdots, v_{k}\right\rangle$ spanned by the vectors $v_{1}, \cdots, v_{k}$, that is, 


$$
\langle v\rangle \oplus T_{x}\left(M_{m_{1}, \cdots, m_{k}}\right)^{\perp}=\left\langle v_{1}, \cdots v_{k}\right\rangle \text { in } C^{\langle n+k\rangle / 2} \text {. }
$$

Let $w_{1}, \cdots, w_{k-1}$ be an orthonormal frame for $T_{x}\left(M_{m_{1}, \cdots, m_{k}}\right)^{1}$. Then $w_{2}$ is given by a linear combination of $v_{1}, \cdots v_{k}$. Thus $f w_{2}$ is tangent to $M_{m_{1}, \cdots, m_{k}}$ and hence

$$
\phi w_{2}=J w_{i}+\eta\left(w_{2}\right) v=J w w_{2} .
$$

Therefore $\phi u_{\iota}$ is tangent to $M_{m_{1}, \cdots, m_{k}}$ for all $\imath=1, \cdots, k-1$. Thus we have

$$
\phi T_{x}\left(M_{m_{1}, \ldots, m_{k}}\right)^{\perp} \subset T_{x}\left(M_{m_{1}, \ldots, m_{k}}\right),
$$

which shows that $M_{m_{1}, \ldots, m_{k}}$ is a generic submanifold of a Sasakian space form $S^{n+k-1}$.

Moreover, we consider an immersion:

$$
S^{m_{1}}\left(r_{1}\right) \times \cdots \times S^{m_{k}}\left(r_{k}\right) \rightarrow S^{n+k-1}, \quad n=\sum_{i=1}^{k} m_{l},
$$

where $r_{1}^{\prime} ; \cdots+r_{k}^{\prime}=1$. Then $S^{m_{1}}\left(r_{1}\right) \times \cdots \times S^{n_{k}}\left(r_{k}\right)$ is a generic submanifold of $S^{n+k-1}$ if $m_{1}, \cdots, m_{k}$ are odd, and it has parallel mean curvature vector and is with fiat normal connection (see [11]). If $m_{1}=m_{2}=\cdots=m_{k}=1$, then $S^{1}\left(r_{1}\right) \times \cdots$ $\times S^{1}\left(r_{k}\right)$ is an anti-invariant submanifold of $S^{2 k-1}$.

\section{§5. Einstein generic submanifolds.}

Let $M$ be an $(n+1)$-dimensional generic submanifold of a $(2 m+1)$-dimensional unit sphere $S^{2 m+1}$ with Sasakian structure. Let $\left\{e_{a}\right\}$ be an orthonormal frame for $T_{x}(M)^{\perp}$. We denote by $A_{a}$ the second fundamental tensor with respect to $e_{a}$, i. e., we put $A_{a}=A_{e_{a}}$. If $M$ is a minimal submanifold of $S^{2 m+1}$, then the Simons' type formula is given by (see [7])

$$
\frac{1}{2} \Delta T=(n+1) T-\sum_{a, b}\left(\operatorname{Tr} A_{a} A_{b}\right)^{2}+\sum_{a, b} \operatorname{Tr}\left[A_{c}, A_{b}\right]^{*}+g(\nabla .4, \nabla A),
$$

$T$ denoting the square of the length of the second fundamental form of $M$, i. e., $T=\sum_{a} \operatorname{Tr} A_{a}^{2}$. We now put

$$
T_{a b}=\operatorname{Tr} A_{a} A_{b}, \quad T_{a}=\operatorname{Tr} A_{a}^{2} .
$$

Since the matrix $\left(T_{a b}\right)$ is symmetric, choosing $\left\{e_{a}\right\}$ suitably, we can diagonalize $\left(T_{a b}\right)$. Then $(5.1)$ reduces to

$$
\frac{1}{2} \Delta T=(n+1) T-\sum_{a} T_{a}^{2}+\sum_{a, b} \operatorname{Tr}\left[A_{a}, A_{b}\right]^{2}+g(\nabla A, \nabla A) .
$$

On the other hand, we have

$$
\sum_{a} T_{a}^{2}=\frac{1}{p} T^{2}+\frac{1}{p} \sum_{a>b}\left(T_{a}-T_{b}\right)^{2}, \quad p=\operatorname{dim} T_{x}(M)^{\perp} .
$$

Consequently, we obtain

$$
\frac{1}{2} \Delta T=(n+1) T-\frac{1}{p} T^{2}-\frac{1}{p} \sum_{a>b}\left(T_{a}-T_{b}\right)^{2}+\sum_{a, b} \operatorname{Tr}\left[A_{a}, A_{b}\right]^{2}+g(\nabla . A, \nabla A) .
$$


In the following we assume that the second fundamental form of $M$ is parallel. Then (5.2) becomes

$$
0=(n+1) T-\frac{1}{p} T^{2}-\frac{1}{p} \sum_{a \rightarrow b}\left(T_{a}-T_{b}\right)^{2}+\sum_{a, b} \operatorname{Tr}\left[A_{a}, A_{b}\right]^{2} .
$$

On the other hand, the Ricci tensor $S$ of $M$ is given by

$$
S(X, Y)=n g(X, Y)-\sum_{\imath} g\left(B\left(X, e_{\imath}\right), B\left(Y, e_{\imath}\right)\right)
$$

where $\left\{e_{i}\right\}$ denotes an orthonormal frame of $M$. Putting $X=Y=\xi$ in (5.4), we find

$$
S(\xi, \xi)=n-\sum_{\imath} g\left(B\left(\xi, e_{\imath}\right), B\left(\xi, e_{\imath}\right)\right)=n-\sum_{\imath} g\left(F e_{\imath}, F e_{\imath}\right)=n-p .
$$

Thus, if $M$ is an Einstein manifold, we have

from which

$$
S(X, Y)=(n-p) g(X, Y) \text {, }
$$

$$
T=\sum_{i, j} g\left(B\left(e_{i}, e_{j}\right), B\left(e_{\imath}, e_{j}\right)\right)=-\sum_{i} S\left(e_{\imath}, e_{\imath}\right)+n(n+1)=(n+1) p .
$$

Thus (5.3) reduces to

$$
0=-\frac{1}{p} \sum_{a>b}\left(T_{a}-T_{b}\right)^{2}+\sum_{a, b} \operatorname{Tr}\left[A_{a}, A_{b}\right]^{2} .
$$

Since we have

$$
-\frac{1}{p} \sum_{a>b}\left(T_{a}-T_{b}\right)^{2} \leqq 0, \quad \sum_{a, b} \operatorname{Tr}\left[A_{a}, A_{b}\right]^{2} \leqq 0,
$$

(5.5) implies that

$$
\begin{gathered}
T_{a}=T_{b} \text { for all } a, b, \\
{\left[A_{a}, A_{b}\right]=0 \text { for all } a, b .}
\end{gathered}
$$

Equation (5.7) shows that the normal connection of $M$ is flat. We now need the following lemmas.

LEMMA 5.1. ([13]). Let $M$ be an n-dimensional submanifold of $S^{n+p}$ with fat normal connection. If the second fundamental form of $M$ is parallel, then the sectional curvature of $M$ is non-negative.

LEMMA 5.2. Let $M$ be a generuc submanifold of a Sasakzan mamijold $\bar{M}$. Then the immersion is full, that is, there is no totally geodesic submamfold $M^{\prime}$ of $\bar{M}$ which contains $M$ as a submanfold.

Proof. Let $V$ be a vector field normal to $M$. If $g(B(X, Y), V)=0$ for any vector fields $X, Y$ tangent to $M$, then putting $Y=-\xi$, we have $g(F X, V)=0$. Since $M$ is a generic submanifold of $\bar{M}$, we can put $X=\phi V$. Then we have $g(F X, V)=g(F \phi V, V)=-g(V, V) \neq 0$, which shows that the immersion is full. 
THEorem A ([11]). Let $M$ be a complete minmal submanufold of dimension $n$ immersed $\mathrm{S}^{m}$ and with non-negative sectional curvature, and suppose that the normal connection of $M$ is flat. If $T$ is constant, then $M$ is a great sphere of $S^{m}$ or a pythagorean product of the form

$$
S^{p_{1}}\left(r_{1}\right) \times \cdots \times S^{\mu_{N}}\left(r_{N}\right), \quad r_{t}=\sqrt{ } p_{t}: \bar{n} \quad(=1, \cdots, N),
$$

and is of essential codimension $N-1$, where $p_{1}, \cdots, p_{N} \geqq 1, p_{1}+\cdots+p_{N}=n$.

We now prove

THEOREM 5.1. Let $M$ be an $(n+1)$-dimensional complete generu minmal submanifold of $S^{2 m+1}$ with parallel second fundamental form. If $M$ is Einstem, then $M$ is

$$
S^{q}(r) \times \cdots \times S^{q}(r) \quad(N \text {-tmes }), \quad r=\sqrt{ } q /(n+1),
$$

where $q$ is an odd number and $2 m-n=N-1, N q=n+1$.

Proof. From the assumption and Lemma 5.1, $M$ has non-negative sectional curvature. Therefore Theorem A implies that $M$ is

$$
S^{p_{1}}\left(r_{1}\right) \times \cdots \times S^{p v}\left(r_{N}\right), \quad r_{t}=\sqrt{p_{t} /(n+1)} \quad(t=1, \cdots, N)
$$

and is of codimension $2 m-n=N-1$ by Lemma 5.2 . Since $\xi$ is tangent to $M$, we see that $p_{1}, \cdots, p_{N}$ are odd numbers and since $M$ is Einstein, we have $p_{1}=\cdots=p_{N}$.

\section{§6. Pseudo-umbilical generic submanifolds.}

Let $M$ be an $(n+1)$-dimensional generic submanifold of a $(2 m+1)$-dimensional Sasakian manifold $\bar{M}$. We now choose an orthonormal frame $\left\{e_{A}\right\}$ of $\vec{M}$ in such a way that $e_{1}=\xi, e_{2}, \cdots, e_{n+1}$ form an orthonormal frame for $M$ and $e_{n+2}, \cdots$, $e_{2 m+1}$ form an orthonormal frame for the normal space $T_{x}(M)^{\perp}$, and moreover that $e_{1}, \cdots, e_{n-p+1}$ form an orthonormal frame for $H_{x}(M), e_{n-p+2}, \cdots, e_{n+1}$ form an orthonormal frame for $\phi T_{x}(M)$ and $\phi e_{n-p+2}=F e_{n-p+2}=e_{n+2}, \cdots, \phi e_{n+1}=F e_{n+1}$ $=e_{2 m+1}$.

Unless otherwise stated, we use the following convention on the ranges of indices: $i, j, k, \cdots=1, \cdots, n+1 ; x, y, z, \cdots=n-p+2, \cdots, n+1 ; \alpha, \beta, \gamma, \cdots=1$, $\cdots, n-p+1$.

If the second fundamental form $B$ of $M$ is of the form

$$
\begin{aligned}
B(X, Y)= & a[g(X, Y)-\eta(X) \eta(Y)] \zeta+\eta(X) F Y+\eta(Y) F X \\
& +\sum_{x} b_{x} g\left(X, e_{x}\right) g\left(Y, e_{x}\right) F e_{x},
\end{aligned}
$$

where $\zeta$ is a unit vector normal to $M$ and $a$ and $b_{x}$ are functions, then $M$ is said to be pseudo-umbilical. In this case we see that 


$$
\begin{aligned}
g( & \left.B(X, Y), F e_{x}\right)=g\left(A_{x} X, Y\right) \\
= & a[g(X, Y)-\eta(X) \eta(Y)] g\left(\zeta, F e_{x}\right)+\eta(X) g\left(Y, e_{x}\right)+\eta(Y) g\left(X, e_{x}\right) \\
& +b_{x} g\left(X, e_{x}\right) g\left(Y, e_{x}\right),
\end{aligned}
$$

where we have written $A_{F e_{x}}$ as $A_{x}$ to simplify the notation. Thus, the second fundamental form $A_{x}$ is represented by a matrix

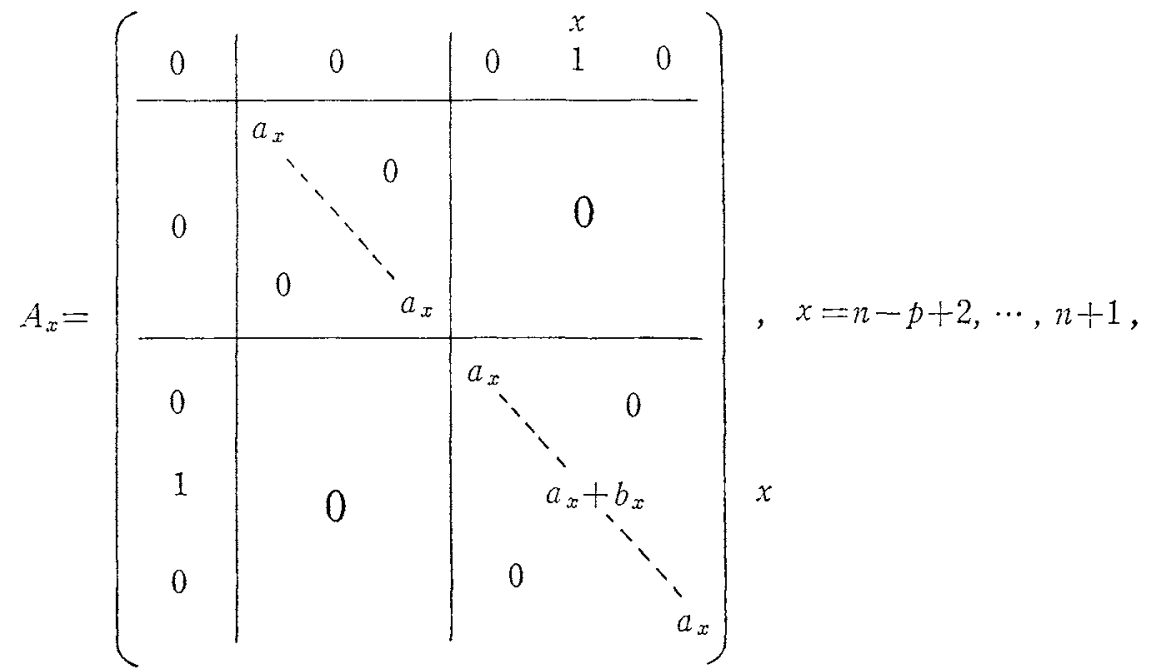

where we have put $a_{x}=a g\left(\zeta, F e_{x}\right)$. On the other hand, from Lemma 2.1, we see that

$$
a_{x}=g\left(A_{x} e_{y}, e_{y}\right)=g\left(A_{y} e_{x}, e_{y}\right)=0 \text { for } x \neq y \text {. }
$$

Therefore, if $p=\operatorname{codim} M \geqq 2$, we have $a_{x}=0$ for all $x$. Thus (6.2) reduces to

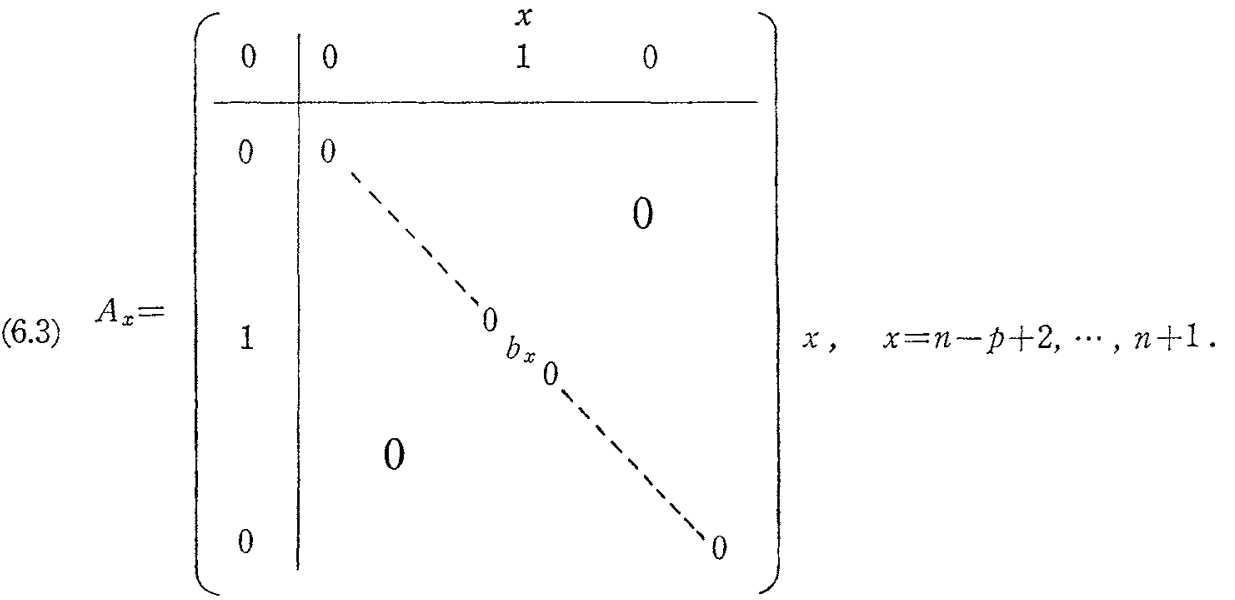


If $p=1$, then (6.2) becomes

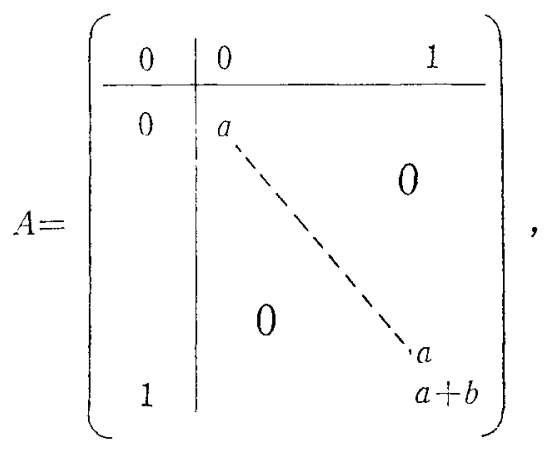

where $A=A_{n+1}$ and $b=b_{n+1}$.

When $p \geqq 2$, the second fundamental form $B$ of a pseudo-umbilical generic submanifold $M$ satisfies

$$
B(X, Y)=a[g(X, Y)-\eta(X) \eta(Y)] \zeta+\eta(X) F Y+\eta(Y) F X
$$

for any vector $X \in H_{x}(M)$ and any vector $Y$ tangent to $M$. Since $a_{x}=a g\left(\zeta, F e_{x}\right)$ $=0$ for all $x$, we have $a=0$ and hence

$$
B(X, Y)=\eta(X) F Y+\eta(Y) F X
$$

for any vector $X \in H_{x}(M)$ and any vector tangent to $M$. From (2.10) and (6.6) we find

$$
\left(\nabla_{X} F\right) Y=-B(X, P Y)=-\eta(X) F P Y-\eta(P Y) F X=0
$$

for any vectors $X$ and $Y$ tangent to $M$. We now consider a distribution $\mathcal{L}: x \rightarrow \mathcal{L}_{x}=\left\{X \in T_{x}(M): F X=0\right\}$. Since we have

$$
F \nabla_{X} Y=D_{X}(F Y)-\left(\nabla_{X} F\right) Y=0
$$

for any $Y \in H(M)$ and any $X \in T(M)$, the distribution $\mathcal{L}$ is parallel and the maximal integral submanifold $M_{1}$ of $\mathcal{L}$ is totally geodesic in $M$. Moreover $M_{1}$ is totally geodesic in $\bar{M}$ and $M_{1}$ is an invariant submanifold of $\bar{M}$. Thus $M_{1}$ is also a Sasakian manifold. Consequently, we have

Proposition 6.1. Let $M$ be an $(n+1)$-dimensional pseudo-umbilical genernc submanifold of a $(2 m+1)$-dimensional Sasakıan manifold $\bar{M}$ with $p \geqq 2$. Then the distribution $\mathcal{L}$ is completely integrable and its maximal integral submanfold $M_{1}$ is totally geodesic, invariant submanifold of $\vec{M}$.

Here we notice that the maximal integral submanifold $M_{2}$ of the distribution $\mathscr{T}: x \rightarrow \mathscr{T}_{x}=\left\{X \in T_{x}(M): P X=0\right.$ and $\left.\eta(X)=0\right\}$ is totally geodesic in $M$. Indeed, if $X, Y$ are vector fields tangent to $M_{2}$ and $Z$ a vector field tangent to $M_{1}$, then we have

$$
g\left(\nabla_{X} Y, Z\right)=-g\left(Y, \nabla_{X} Z\right)=0
$$


because of the fact that $\nabla_{X} Z$ is tangent to $M_{1}$ and $Z$ is normal to $M_{2}$. Thus $\nabla_{X} Y$ is tangent to $M_{2}$ and hence $M_{2}$ is totally geodesic in $M$.

From Propositions 3.1 and 6.1 we have

Proposition. 6.2. Let $M$ be an $(n+1)$-dimensional pseudo-umbilical generuc submantold of a $(2 m+1)$-dimensional Sasakian manifold $\bar{M}$ with $p \geqq 2$. Then $M$ is locally a Remannian direct product of the form $M_{1} \times M_{2}$, where $M_{1}$ is an $(n-p+1)$-dinensional totally geodesic invariant submanifold of $\bar{M}$ and $M_{2}$ a p-dimensional anti-invariant submanifold of $\bar{M}$ normal to $\xi$.

THEOREM 6.1. Let $M$ be an $(n+1)$-dimensional pseudo-umbilical generic submanffold of a $(2 m+1)$-dimensional Sasakian manifold $\bar{M}$ with vanishing contact Bochner curvature tensor. If $p \geqq 4$, then $M$ is locally a Riemannan direct product of the form $M_{1} \times M_{2}$, where $M_{1}$ is $a(n-p+1)$-dimensional totally geodesic invarlant submanifold of $\bar{M}$ and has vanishing contact Bochner curvature tensor and $M_{2}$ is a p-dimensional conformally flat, anti-invariant submanifold of $\bar{M}$ normal to $\xi$.

Proof. From Proposition 6.2, $M$ is locally of the form $M_{1} \times M_{2}$, where $M_{1}$ is a totally geodesic, invariant submanifold of $\bar{M}$ and $M_{2}$ an anti-invariant submanifold of $\bar{M}$ normal to $\xi$. Since $M_{1}$ is totally geodesic, the contact Bochner curvature tensor of $M_{1}$ vanishes (see [2]).

Since $M_{2}$ is totally geodesic in $M$, the second fundamental form of $M_{2}$ in $\bar{M}$ is given by $B(X, Y)$ for any vector fields $X$ and $Y$ tangent to $M_{2}$. From (2.9) we have

$$
\phi B(X, Y)=-A_{F X} Y+g(X, Y) \xi
$$

for any vector fields $X$ and $Y$ tangent to $M_{2}$. Let $X, Y, Z$ and $W$ be vector fields tangent to $M_{2}$. Then (6.7) implies

$$
\begin{aligned}
& g(B(X, W), B(Y, Z))-g(B(X, Z), B(Y, W)) \\
= & g\left(A_{F X} W, A_{F Y} Z\right)-g\left(A_{F X} Z, A_{F Y} W\right)+g(X, Z) g(Y, W)-g(Y, Z) g(X, W) .
\end{aligned}
$$

Put $X=\sum_{x} X^{x} e_{x}$ and $W=\sum_{x} W^{x} e_{x}$. Then (6.3) implies

$$
\begin{aligned}
A_{F X} W & =\sum_{x, y} X^{x} W^{y} A_{x} e_{y}=\sum_{x} X^{x} W^{x} A_{x} e_{x} \\
& =\sum_{x} X^{x} W^{x}\left(b_{x} e_{x}+\xi\right)=\sum_{x} X^{x} W^{x} b_{x} e_{x}+g(X, W) \xi .
\end{aligned}
$$

From (6.8) and (6.9) we have

$$
g(B(X, W), B(Y, Z))-g(B(X, Z), B(Y, W))=0 .
$$

Consequently, Lemma 9.1 of $\left[12 ;\right.$ p. 147] implies that $M_{2}$ is conformally flat. This proves our assertion.

In the sequel, we assume that $M$ is an $(n+1)$-dimensional pseudo-umbilical generic submanifold of a Sasakian space form $\bar{M}^{2 m+1}(k)$ with $p \geqq 2$. We assume 
that $M$ has non-zero parallel mean curvature vector $\mu$. Then (1.12) implies

$$
\frac{1}{4}(k-1)[g(F Y, \mu) g(F X, V)-g(F X, \mu) g(F Y, V)]=g\left(\left[A_{V}, A_{\mu}\right] X, Y\right) .
$$

By a straightforward computation, we can see that this becomes

$$
\frac{1}{4}(k+3)[g(F Y, \mu) g(F X, V)-g(F X, \mu) g(F Y, V)]=0 \text {. }
$$

Since $p \geqq 2$, we have $k=-3$. In the following, we prove that $M_{2}$ has non-zero $\eta$-parallel mean curvature vector (for the $\eta$-parallel mean curvature vector, see [12; p. 124]). Since $M_{2}$ is totally geodesic in $M$, the mean curvature vector $\mu^{\prime}$ of $M_{2}$ in $\bar{M}$ is equal to $\frac{n+1}{p}-\mu$. Let $A^{\prime}$ be the second fundamental form of $M_{2}$ in $\bar{M}$ and $D^{\prime}$ be the operator of covariant differentiation of the normal bundle of $M_{2}$ in $\bar{M}$. For any vector field $X$ tangent to $M_{2}$ we obtain

$$
D^{\prime}{ }_{X} \mu^{\prime}=\bar{\nabla}_{X} \mu^{\prime}+A^{\prime}{ }_{\mu^{\prime}} X=-A_{\mu^{\prime}} X+{ }_{p}^{n+1} D_{X} \mu+A^{\prime}{ }_{\mu^{\prime}} X \text {. }
$$

Let $Y$ be a vector field tangent to $M_{1}$. Then we have

$$
g\left(D^{\prime}{ }_{X} \mu^{\prime}, Y\right)=-g\left(A_{\ell^{\prime}} X, Y\right)=-g\left(B(X, Y), \mu^{\prime}\right)=-\eta(Y) g\left(\mu^{\prime}, F X\right),
$$

where we have used (6.5). If $\eta(Y)=0$, then we have $g\left(D^{\prime}{ }_{x} \mu^{\prime}, Y\right)=0$. Let $N$ be a vector field normal to $M$. Then we have

$$
g\left(D^{\prime}{ }_{X} \mu^{\prime}, N\right)=\frac{n+1}{p} g\left(D_{X} \mu, N\right)=0
$$

because of $D_{X} \mu=0$. Therefore $M_{2}$ has non-zero $\eta$-parallel mean curvature vector, that is, the mean curvature vector $\mu^{\prime}$ of $M_{2}$ in $\bar{M}$ satisfies $g\left(D^{\prime}{ }_{x} \mu^{\prime}, Y\right)$ $=0$ for any vector field $X$ tangent to $M_{2}$ and any vector field $Y$ normal to $M_{2}$ in $\bar{M}$ such that $\eta(Y)=0$. Therefore we have

Proposition 6.3. Let $M$ be an $(n+1)$-dimensional pseudo-umbilical generic submanifold of a Sasakian space form $\bar{M}^{2 m+1}(k)$ with non-zero parallel mean curvature vector. If $p \geqq 2$, then $k=-3$ and $M$ is locally a Riemannian direct product $M_{1} \times M_{2}$, where $M_{1}$ is totally geodesic invariant submanifold of $\bar{M}^{2 m+1}(-3)$ and $M_{2}$ an anti-invariant submanfold of $\bar{M}^{2 m+1}(-3)$ with non-zero $\eta$-parallel mean curvature vector.

We denote by $E^{2 m+1}(-3)$ the Sasakian space form with constant $\phi$-sectional curvature -3 with standard Sasakian structure in a Euclidean space (cf. [12]).

From Proposition 6.3 and Theorem 6.1 of $[12 ;$ p. 143], we have

THEOREM 6.2. Let $M$ be an $(n+1)$-dimensional complete pseudo-umbilical genernc submanifold of a simply connected complete Sasakian space form $\bar{M}^{2 m+1}(k)$ with 
non-zero parallel mean curvature vector. If $p \geqq 2$, then $M$ is a pythagorean product of the form

$$
E^{n-p+1}(-3) \times S^{1}\left(r_{1}\right) \times \cdots \times S^{1}\left(r_{p-s}\right) \times R^{s} \text { in } E^{2 m+1}(-3)
$$

or

$$
E^{n-p+1}(-3) \times S^{1}\left(r_{1}\right) \times \cdots \times S^{1}\left(r_{p}\right) .
$$

Proof. By Proposition 6.3, $M$ is $M_{1} \times M_{2}$ and $k=-3$. Thus we have $\bar{M}^{2 m+1}(-3)=E^{2 m+1}(-3)$. Since $M_{1}$ is a totally geodesic invariant submanifold of $E^{2 m+1}(-3)$, we have $M_{1}=E^{n-p+1}(-3)$.

In the following, we study $M_{2}$ in $E^{2 m+1}(-3)$. First of all, we consider the second fundamental form $A^{\prime}$ of $M_{2}$ in $E^{2 m+1}(-3)$. Let $X$ be a vector field tangent to $M_{2}$ and $V$ a vector field tangent to $M_{1}$. Then $V$ is normal to $M_{2}$. Thus we have

$$
\bar{\nabla}_{X} V=-A^{\prime}{ }_{V} X+D^{\prime}{ }_{X} V=\nabla_{X} V+B(X, V) .
$$

Since we have $\nabla_{X} V \in T\left(M_{1}\right)$, we see that $A^{\prime}{ }_{V} X=0$. Let $N$ be a vector field normal to $M$. Then we have

$$
\bar{\nabla}_{X} N=-A^{\prime}{ }_{N} X+D^{\prime}{ }_{X} N=-A_{N} X+D_{X} N \text {, }
$$

from which we obtain $g\left(A^{\prime}{ }_{N} X, Y\right)=g\left(A_{N} X, Y\right)$, where $Y$ is a vector field tangent to $M_{2}$. From this and (6.3) we have

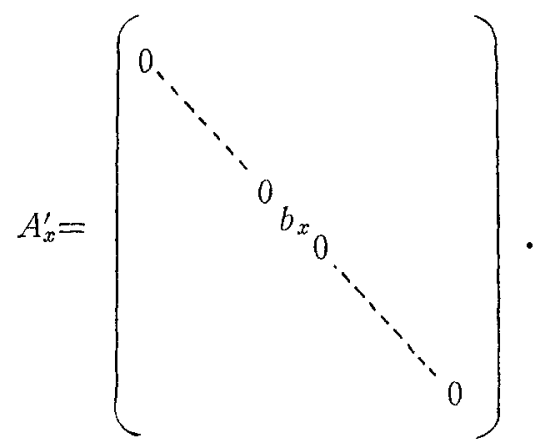

Thus we see that the second fundamental forms of $M_{2}$ in $E^{2 m+1}(-3)$ are commutative.

Let $N$ be a vector field normal to $M_{2}$ in $E^{2 m+1}(-3)$. We put

$$
\phi N=t N+f N,
$$

where $t N$ is the tangential part and $f N$ the normal part of $\phi N$ respectively. Then $f$ defines an $f$-structure in the normal bundle of $M_{2}$ (see $[12 ;$ p. 122]). Thus we have

from which

$$
\left(D^{\prime}{ }_{X} f\right) N=-B(X, t N)-\phi A^{\prime}{ }_{N} X
$$

$$
g\left(\left(D^{\prime}{ }_{X} f\right) N, V\right)=-g\left(A^{\prime} X, t N\right)+g\left(A^{\prime}{ }_{N} X, t V\right)
$$


where $V$ is a vector field normal to $M_{2}$. If $N$ is tangent to $M_{1}$, then we have $t N=0$ and $A^{\prime}{ }_{N} X=0$. If $V$ is tangent to $M_{1}$, we have $t V=0$ and $A^{\prime}{ }_{V} X=0$. Next we suppose that $N$ and $V$ are normal to $M$. Then Lemma 2.1 implies that $A^{\prime}{ }_{N}(t V)=A^{\prime}{ }_{V}(t N)$. Consequently, we obtain $\left(D^{\prime}{ }_{x} f\right) N=0$, which shows that the $f$-structure of the normal bundle is parallel. Thus Theorem 6.1 of $[12 ;$ p. 143] implies that $M_{2}$ is of the form

or

$$
S^{1}\left(r_{1}\right) \times \cdots \times S^{\perp}\left(r_{p-s}\right) \times R^{s}
$$

$$
S^{1}\left(r_{1}\right) \times \cdots \times S^{1}\left(r_{p}\right) .
$$

Therefore we have our assertion.

\section{§7. Pseudo-umbilical hypersurfaces.}

Let $M$ be a pseudo-umbilical hypersurface of a Sasakian manifold $\bar{M}$. Then, from (6.1), we see that the second fundamental form $A$ of $M$ is of the form

$$
A X=a[X-\eta(X) \xi]+b u(X) U+\eta(X) U+u(X) \xi
$$

for any vector field $X$ tangent to $M, a$ and $b$ being functions.

The notation of pseudo-umbilical hypersurfaces of Sasakian manifolds corresponds to that of $\eta$-umbilical real hypersurfaces of Kaehlerian manifolds (cf. [4]). A real hypersurface $N$ of a Kaehlerian manifold $\bar{N}$ is said to be $\eta$-umbilical if the second fundamental form $H$ of $N$ is of the form $H X=\alpha X+\beta h(X) V$, where $V$ is a unit vector field normal to $N$ and $h$ is a dual 1-form of $V$, and $\alpha, \beta$ are functions.

We now prove the following

PRoPosition 7.1. Let $\bar{M}$ be a regular Sasakzan manfold and $M$ be a hypersurface of $\bar{M}$ tangent to $\xi$, and let $\bar{N}$ be a Kaehlertan manfold and $N$ be a real hypersurface of $\bar{N}$ such that the diagram

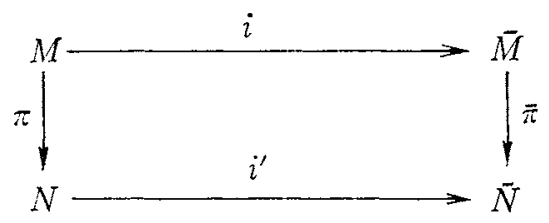

commutes and the immersion $\imath$ is a diffeomorphisin on the fibres. Then $M$ is pseudo-umbilical if and only if $N$ is $\eta$-umbilical.

Proof. Let $X$ and $Y$ be vector fields tangent to $N$. We denote by * the horizontal lift with respect to $\eta$. Then we have (cf. [12]), $(G(H X, Y))^{*}=$ $g\left(A X^{*}, Y^{*}\right), G$ being the metric tensor field of $N$. If $N$ is $\gamma_{\text {-umbilical, then }}$ we find $g\left(A X^{*}, Y^{*}\right)=\alpha g\left(X^{*}, Y^{*}\right)+\beta u\left(X^{*}\right) u\left(Y^{*}\right)$, where we have used $V^{*}=U$. 
Thus we have $g\left(A \phi^{2} X, \phi^{2} Y\right)=\alpha g\left(\phi^{2} X, \phi^{2} Y\right)+\beta u\left(\phi^{2} X\right) u\left(\phi^{2} Y\right)$ for any vector fields $X, Y$ tangent to $M$. From this we have (7.1). The converse is also true by virtue of (7.1).

Let $M$ be a pseudo-umbilical hypersurface of a Sasakian space form $\bar{M}^{2 n+1}(k)$. Then (7.1) implies

$$
\begin{aligned}
\left(\nabla_{Y} A\right) X= & (Y a)[X-\eta(X) \xi]+(Y b) u(X) U+a[-g(P Y, X) \xi-\eta(X) P Y] \\
& +b[g(P A Y, X) U+u(X) P A Y] \\
& +g(P Y, X) U+\eta(X) P A Y+g(P A Y, X) \xi+u(X) P Y
\end{aligned}
$$

for any vector fields $X$ and $Y$ tangent to $M$. From this we have

$$
\begin{aligned}
\left(\nabla_{X} A\right) Y-\left(\nabla_{Y} A\right) X= & (X a)[Y-\eta(Y) \xi]-(Y a)[X-\eta(X) \xi] \\
& +(X b) u(Y) U-(Y b) u(X) U+a[2 g(P Y, X) \xi \\
& -\eta(Y) P X+\eta(X) P Y]+b[g(P A X, Y) U \\
& -g(P A Y, X) U+u(Y) P A X-u(X) P A Y] \\
& +2 g(P X, Y) U+\eta(Y) P A X-\eta(X) P A Y \\
& +g(P A X, Y) \xi-g(P A Y, X) \xi+u(Y) P X-u(X) P Y .
\end{aligned}
$$

Combining this with (1.11), we find

$$
\begin{aligned}
& \frac{1}{4}(k+3)[g(P Y, Z) u(X)-g(P X, Z) u(Y)-2 g(P X, Y) u(Z)] \\
= & (X a)[g(Y, Z)-\eta(Y) \eta(Z)]-(Y a)[g(X, Z)-\eta(X) \eta(Z)]+(X b) u(Y) u(Z) \\
& -(Y b) u(X) u(Z)+a[2 g(P Y, X) \eta(Z)-\eta(Y) g(P X, Z)+\eta(X) g(P Y, Z)] \\
& +b[g(P A X, Y) u(Z)-g(P A Y, X) u(Z)+g(P A X, Z) u(Y)-g(P A Y, Z) u(X)] \\
& +\eta(Y) g(P A X, Z)-\eta(X) g(P A Y, Z)+\eta(Z) g(P A X, Y)-\eta(Z) g(P A Y, X)
\end{aligned}
$$

for any vector fields $X, Y$ and $Z$ tangent to $M$.

Putting $Y=U$ in (7.2) and using (7.1), we have

$$
\begin{aligned}
{\left[a b+\frac{1}{4}(k+3)\right] g(P X, Z)=} & -u(Z) X(a+b)+(U b) u(X) u(Z) \\
& +(U a)[g(X, Z)-\eta(X) \eta(Z)] .
\end{aligned}
$$

Moreover, putting $Z=U$ in this equation, we find $X(a+b)=u(X) U(a+b)$, from which

$$
\left[a b+\frac{1}{4}(k+3)\right] g(P X, Z)=(U a)[g(X, Z)-\eta(X) \eta(Z)]-(U a) u(X) u(Z) .
$$

Since $P$ is skew-symmetric, we have

$$
\left[a b+\frac{1}{4}(k+3)\right] g(P X, Z)=0 .
$$


If $n \geqq 2$, then (7.3) implies that $a b=-\frac{1}{4}(k+3)$. Suppose that the ambient manifold $\bar{M}^{2 n+1}(k)$ is $S^{2 n+1}$. Then we have $a b=-1$. Since $g(A U, U)=a+b$, we obtain

$$
X(a+b)=g\left(\left(\nabla_{X} A\right) U, U\right)=(U(a+b)) u(X),
$$

where we have used $P A U=0$ and (2.5). We put $\beta=U(a+b)$. 'Then we have

$$
\begin{aligned}
& X Y(a+b)=(X \beta) u(Y)+\beta a g(P X, Y)+\beta u\left(\nabla_{X} Y\right), \\
& Y X(a+b)=(Y \beta) u(X)+\beta a g(P Y, X)+\beta u\left(\nabla_{Y} X\right), \\
& {[X, Y](a+b)=\beta u([X, Y]) .}
\end{aligned}
$$

Since $R(X, Y)(a+b)=0$, we find

$$
0=(X \beta) u(Y)-(Y \beta) u(X)+2 \beta a g(P X, Y) \text {. }
$$

Since $n \geqq 2$ and $a \neq 0$, we have $\beta=0$ and hence (7.4) implies that $X(a+b)=0$ for any vector field $X$ tangent to $M$. Therefore $a+b$ is a constant. From this and $a b=-1, a$ and $b$ are both constant. On the other hand, the second fundamental form $A$ is represented in the following matrix form, for an orthonormal frame $e_{1}, \cdots, e_{2 n}$ such that $e_{2 n-1}=U$ and $e_{2 n}=\xi$,

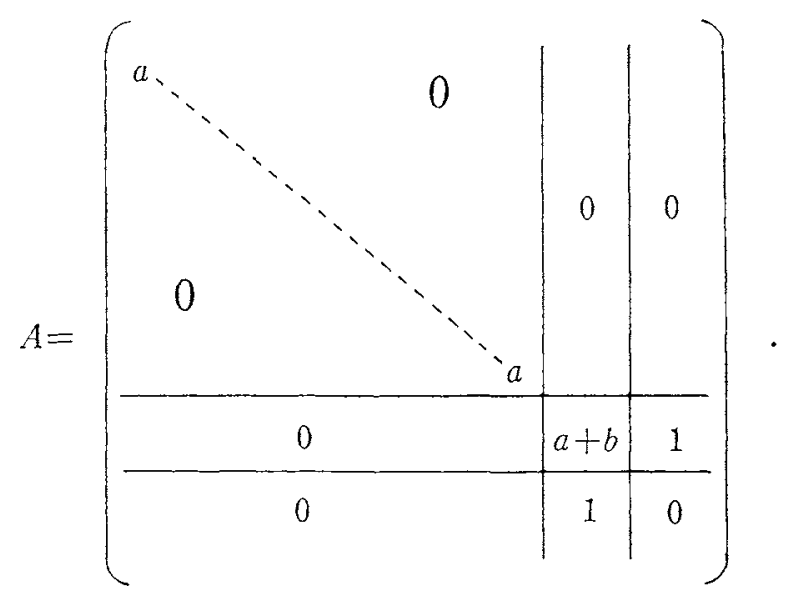

We consider the matrix $\left(\begin{array}{cc}a+b & 1 \\ 1 & 0\end{array}\right)$. Then the eigenvalue $\lambda$ of this matrix satisfies $\lambda^{2}-(a+b) \lambda+a b=0$. Since $a b=-1$, we have $\lambda=a$ or $\lambda=b$. Therefore, the principal curvatures of $M$ are $a$ and $b$. The multiplicity of $a$ is $2 n-1$ and that of $b$ is 1 . Consequently, we have

LEMMA 7.1. Let $M$ be a pseudo-umbilical hypersurface of $S^{2 n+1}(n \geqq 2)$. Then $M$ has two constant principal curvatures with multiplicities $2 n-1$ and 1 respec- 
tively.

From Lemma 7.1 and a well known theorem (cf. [6]), we have

THEOREM 7.1. Let $M$ be a compact pseudo-umbilical hypersurface of $S^{2 n+1}(n \geqq 2)$. Then $M$ is congruent to

$$
S^{2 n-1}\left(r_{1}\right) \times S^{1}\left(r_{2}\right), \quad r_{1}^{2}+r_{2}^{2}=1
$$

\section{§ 8. A characterization of Sasakian space form.}

First of all, we define an axiom, which will be called a $P$-axiom. A Sasakian manifold $M$ of dimension $2 n+1$ is said to satisfy the $P$-axiom if for each $x \in M$ and each $2 n$-dimensional subspace $S$ of $T_{x}(M), \xi \in S$, there exists a pseudoumbilical hypersurface $N$ such that $T_{x}\left(N^{*}\right)=S, \quad x \in N$ and $g(A U, U)=a+b=$ constant.

The purpose of this section is to prove the following

THEOREM 8.1. If a $(2 n+1)$-dimensional Sasakian manfold $M(n \geqq 2)$ satisfies the P-axiom, then $M$ is a Sasakian space form.

Proof. Let $R$ be the Riemannian curvature tensor of $M$. Then $R$ satisfies

$$
R(X, Y) \phi=\phi R(X, Y)-\phi X \wedge Y-X \wedge \phi Y
$$

where $(X \wedge Y) Z=g(Y, Z) X-g(X, Z) Y$.

Let $x$ be an arbitrary point of $M$ and $C$ a unit vector in $T_{x}(M)$ such that $\eta(C)=0$. Let $S$ be a $2 n$-dimensional subspace of $T_{x}(M)$ orthogonal to $C$. By the $P$-axiom, there exists a pseudo-umbilical hypersurface $N$ such that $T_{x}(M)=S$. From (8.1) we have

$$
\begin{aligned}
g(R(C, \phi C) C, X) & =g(R(C, \phi C) \phi C, \phi X) \\
& =g(R(C, U) U, P X)=g(R(P X, U) U, C)
\end{aligned}
$$

for any vector field $X$ tangent to $N$ such that $u(X)=\eta(X)=0$. On the other hand, equation of Codazzi is given by

$$
g(R(X, Y) Z, C)=g\left(\left(\nabla_{X} A\right) Y, Z\right)-g\left(\left(\nabla_{Y} A\right) X, Z\right)
$$

for any vector fields $X, Y$ and $Z$ tangent to $N$. By a similar computation as that done in $\$ 7$, we find, using (8.2),

$$
g(R(C, \phi C) C, X)=(P X)(a+b) .
$$

Since $a+b$ is a constant, we obtain $g(R(C, \phi C) C, X)=0$. Therefore $R(C, \phi C) C$ is proportional to $\phi C=-U$. From this our theorem follows by virtue of the 
following lemma.

Lemma 8.1. ([9]). A (2n+1)-dimensional ( $n \geqq 2)$ Sasakian manifold $M$ is a Sasakian space form if and only if $R(X, \phi X) X$ is proportional to $\phi X$ for any vector field $X$ of $M$ such that $\eta(X)=0$.

\section{§9. Pseudo-Einstein hypersurfaces.}

Let $M$ be a $2 n$-dimensional hypersurface of $S^{2 n+1}$. Then the Ricci tensor $S$ of $M$ is given by

$$
S(X, Y)=(2 n-1) g(X, Y)+H g(A X, Y)-g(A X, A Y),
$$

$H$ denoting the mean curvature of $M$. If the Ricci tensor $S$ of $M$ is of the form

$$
S\left(\phi^{2} X, \phi^{2} Y\right)=a g\left(\phi^{2} X, \phi^{2} Y\right)+b u\left(\phi^{2} X\right) u\left(\phi^{2} Y^{*}\right)
$$

for any vector fields $X$ and $Y$ tangent to $M, a$ and $b$ being constant, then $M$ is called a pseudo-Einstem hypersurface of $S^{2 n+1}$. Equation (9.2) is equivalent to

$$
\begin{aligned}
S(X, Y)= & a[g(X, Y)-\eta(X) \eta(Y)]+b u(X) u(Y) \\
& +\eta(X) S(\xi, Y)+\eta(Y) S(\xi, X)-\eta(X) \eta(Y) S(\xi, \xi) .
\end{aligned}
$$

We notice here that $S(\xi, \xi)=2 n-2$.

The purpose of this section is to determine complete pseudo-Einstein hypersurface of $S^{2 n+1}$.

If $M$ is a pseudo-Einstein hypersurface of $S^{2 n+1}$, from (9.1) and (9.3), we have

$$
\begin{aligned}
a & {[g(X, Y)-\eta(X) \eta(Y)]+b u(X) u(Y)+\eta(X) S(\xi, Y)+\eta(Y) S(\xi, X) } \\
& -\eta(X) r_{Y}(Y) S(\xi, \xi)=(2 n-1) g(X, Y)+H g(A X, Y)-g(A X, A Y) .
\end{aligned}
$$

In the following, we assume that $n \geqq 3$. We can choose a local field of orthonormal frames $e_{1}, \cdots, e_{2 n-1}, e_{2 n}, e_{2 n+1}$ in $S^{2 n+1}$ in such a way that, restricted to $M, e_{1}, \cdots, e_{2 n}$ are tangent to $M$ and $e_{2 n-1}=\xi, e_{2 n}=U, e_{2 n+1}=\dot{\phi} e_{2 n}=C$. Then if we choose $e_{1}, \cdots, e_{2 n-2}$ suitably, the second fundamental form $A$ is represented by a matrix of the form 
(9.5)

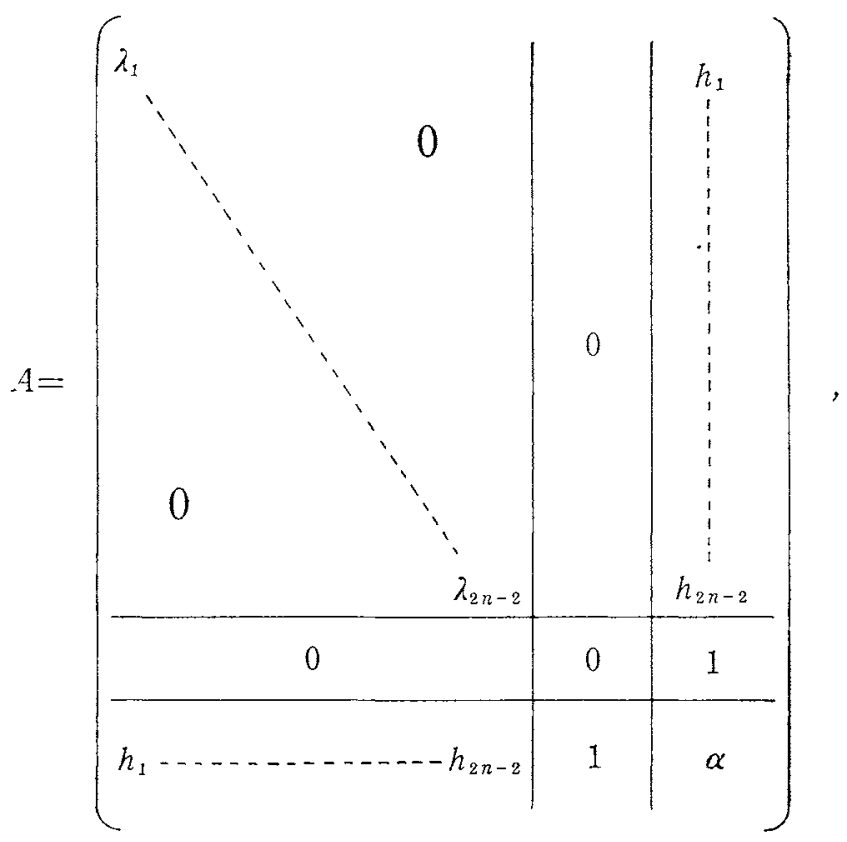

where we have put $h_{\imath}=g\left(A U, e_{2}\right), \imath=1, \cdots, 2 n-2, \alpha=g(A U, U)$. Then from (9.4) and (9.5) we have

$$
\begin{gathered}
g\left(A e_{\imath}, A e_{\jmath}\right)=0 \text { for } \imath \neq \jmath, \imath, \jmath=1, \cdots, 2 n-2, \\
H g\left(A e_{\imath}, U\right)-g\left(A e_{\imath}, A U\right)=0 \text { for } \imath=1, \cdots, 2 n-2 .
\end{gathered}
$$

From these equations we have

$$
\begin{aligned}
& h_{i} h_{j}=0, \quad \imath \neq \jmath, \imath, \jmath=1, \cdots, 2 n-2, \\
& h_{i}\left(H-\lambda_{\imath}-\alpha\right)=0, \quad \imath=1, \cdots, 2 n-2 .
\end{aligned}
$$

Equation (9.6) shows that at most one $h_{\imath}$ does not vanish. Thus we can assume that $h_{\imath}=0$ for $\imath=2, \cdots, 2 n-2$. Then (9.7) implies

Lemma 9.1. Let $M$ be a pseudo-Einstem hypersurface of $S^{2 n+1}$. Then we have $H=\lambda_{1}+\alpha$ or $h_{1}=0$.

On the other hand, from (9.4), we obtain

$$
\begin{gathered}
a=(2 n-1)+H \lambda_{2}-\lambda_{\imath}^{3}, \quad \imath=2, \cdots, 2 n-2, \\
a=(2 n-1)+H \lambda_{1}-\lambda_{1}^{3}-h_{1}^{2}, \\
a+b=(2 n-2)+H \alpha-\alpha^{2}-h_{1}^{2} .
\end{gathered}
$$


We now suppose that $H=\lambda_{1}+\alpha$. Then (9.9) and (9.10) imply that $b=-1$. Thus, for any vector fields $X, Y$ tangent to $M$ such that $\eta(X)=0, r(Y)=0$, we have

$$
a g(X, Y)-u(X) u(Y)=(2 n-1) g(X, Y)+H g(A X, Y)-g(A X, 1 Y) .
$$

We now take a new local field of orthonormal frames $e_{1}, \cdots e_{2 n}$ of $W$ such that $e_{2 n}=\xi$ for which the second fundamental form $A$ is represented by a matrix of the form

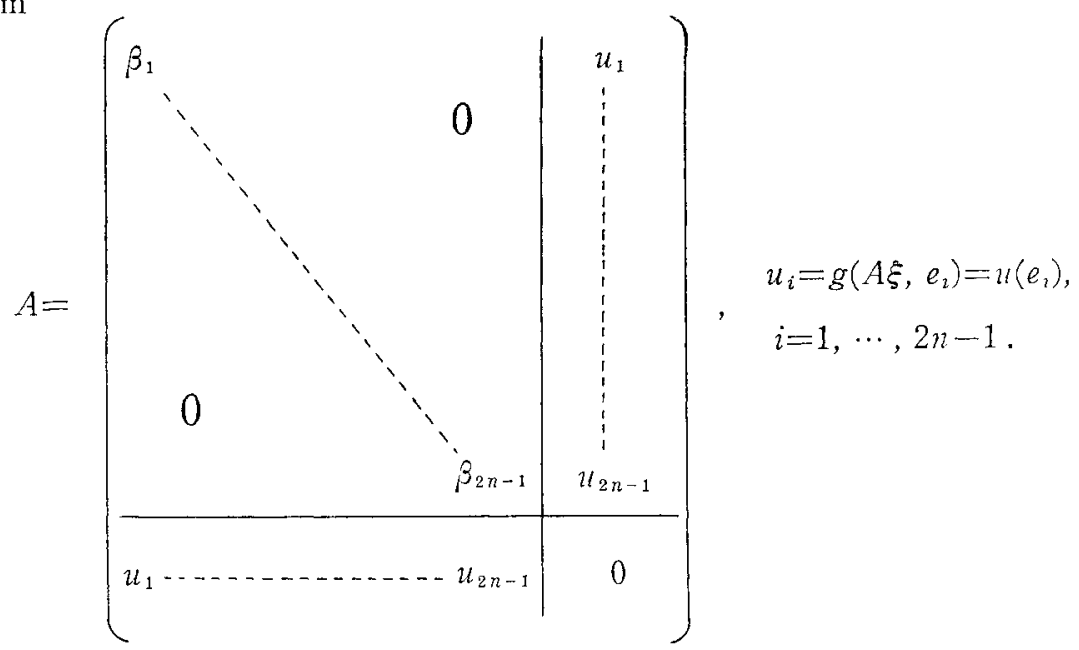

From (9.11) and (9.12) we have

from which

$$
a-u\left(e_{1}\right) u\left(e_{2}\right)=(2 n-1)+H \beta_{i}-\beta_{i}^{2}-u\left(e_{2}\right) u\left(e_{2}\right),
$$

$$
a=(2 n-1)+H \beta_{i}-\beta_{i}^{2}, \quad \imath=1, \cdots, 2 n-1 .
$$

Therefore we see that each $\beta_{2}$ satisfies the quadratic equation

$$
t^{2}-H t+a-(2 n-1)=0 .
$$

We now prepare some lemmas. We put

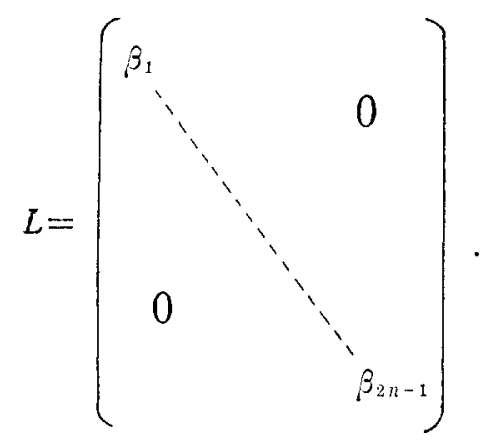


LeMMA 9.2. If $\beta_{1}=\cdots=\beta_{2 n-1}=\beta$ at every point of $M$, then $M$ is totally contact-umbilical.

Proof. By the assumption we have

$$
A e_{\imath}=\beta e_{i}+u\left(e_{i}\right) \xi, \quad \imath=1, \cdots, 2 n-1 .
$$

On the other hand, any vector field $X$ tangent to $M$ is of the form

$$
X=\sum_{i-1}^{2 n-1} g\left(X, e_{\imath}\right) e_{i}+\eta(X) \xi
$$

Therefore, we obtain

$$
A X=\beta X+\eta(X) U+[u(X)-\beta \gamma(X)] \xi .
$$

Thus (1.1) and (1.13) show that $M$ is totally contact-umbilical.

LeMin 9.3. If $A U=\alpha U+\xi$, then $\alpha$ is a constant.

Proof. From the assumption we have

$$
\left(\nabla_{X} A\right) U+A P A X=\left(\nabla_{X} \alpha\right) U+P A X+P X .
$$

From this and equation of Codazzi we have

$$
\begin{aligned}
g & \left(\left(\nabla_{X} A\right) U, Y\right)-g\left(\left(\nabla_{Y} A\right) U, X\right) \\
= & \left(\nabla_{X} \alpha\right) u(Y)+\alpha g(P A X, Y)+g(P X, Y)-g(A P A X, Y) \\
& -\left(\nabla_{Y} \alpha\right) u(X)-\alpha g(P A Y, X)-g(P Y, X)+g(A P A Y, X)=0 .
\end{aligned}
$$

Thus we have

$\left(\nabla_{X} \alpha\right) u(Y)-\left(\nabla_{X} \alpha\right) u(X)+\alpha g((P A+A P) X, Y)+2 g(P X, Y)-2 g(A P A X, Y)=0$.

Putting $X=U$ in this equation, we obtain

Therefore we have

$$
\left(\nabla_{U} \alpha\right) u(Y)=\left(\nabla_{Y} \alpha\right)
$$

$$
\alpha g((P A+A P) X, Y)+2 g(P X, Y)-2 g(A P A X, Y)=0 .
$$

Put $\nabla_{I} \alpha=\gamma$. Then $\nabla_{X} \alpha=\gamma u(X)$ and $\nabla_{Y} \alpha=\gamma u(Y)$ and consequently we have

$$
\nabla_{X} \nabla_{Y} \alpha=\left(\nabla_{X} \gamma\right) u(Y)+\gamma g(Y, P A X)+\gamma g\left(U, \nabla_{X} Y\right) \text {, }
$$

from which

$$
R(X, Y) \alpha=\left(\nabla_{X} \gamma\right) u(Y)-\left(\nabla_{Y} \gamma\right) u(X)+\gamma g((P A+A P) X, Y)=0 .
$$

Putting $X=U$ or $Y=U$ in this equation, we find $\left(\nabla_{U} \gamma\right) u(Y)=\nabla_{Y} \gamma$ and $\left(\nabla_{U} \gamma\right) u(X)$ $=\nabla_{x} \gamma$. Thus we have

$$
\gamma g((P A+A P) X, Y)=0 .
$$

If we assume that $P A+A P=0$, then (9.16) implies 
from which

$$
g(P X, Y)=g(A P A X, Y),
$$

$$
g(P X, P X)=g(P A X, A P X)=-g(P A X, P A X) .
$$

Thus we have $P X=0$. This is a contradiction to the fact that $n \geqq 3$. Consequently we have $\gamma=0$, i. e., $\nabla_{U} \alpha=0$ and hence $\left(\nabla_{U} \alpha\right) u(X)=\left(\nabla_{X} \alpha\right)=0$ for any vector field $X$ tangent to $M$. This shows that $\alpha$ is a constant.

LEMMA 9.4. $\operatorname{rank} L>1$ at some pount of $M$.

Prooj. We assume that $\beta_{1}=\cdots=\beta_{2 n-2}=0$ and put $\beta_{z_{n-1}}=\beta$. Then we see that $A e_{\imath}=-u\left(e_{\imath}\right) \xi$ for $\imath=1, \cdots, 2 n-2$. From this we have

$$
g\left(\left(\nabla_{e_{\imath}} A\right) e_{j}, e_{k}\right)=u\left(e_{k}\right) g\left(P e_{2}, e_{j}\right)+u\left(e_{j}\right) g\left(P e_{\imath}, e_{k}\right),
$$

where $\imath, l, k=1, \cdots, 2 n-2$. Therefore the equation of Codazzi implies

$$
2 u\left(e_{k}\right) g\left(P e_{2}, e_{j}\right)+u\left(e_{j}\right) g\left(P e_{i}, e_{k}\right)-u\left(e_{i}\right) g\left(P e_{j}, e_{k}\right)=0 \text {. }
$$

Putting here $y=k$, we find

from which

$$
u\left(e_{j}\right) g\left(e_{j}, P e_{\imath}\right)=0
$$

$$
\begin{aligned}
& \sum_{i=1}^{2 n-2} u\left(e_{\jmath}\right) g\left(e_{i}, P e_{2}\right) g\left(e_{i}, P e_{2 n-1}\right) \\
& \quad=u\left(e_{j}\right) g\left(P e_{j}, P e_{2 n-1}\right)=-u\left(e_{j}\right) u\left(e_{j}\right) u\left(e_{2 n-1}\right)=0 .
\end{aligned}
$$

Therefore we have $u\left(e_{j}\right)=0, \jmath=1, \cdots, 2 n-2$ or $u\left(e_{2 n-1}\right)=0$.

Let $u\left(e_{3}\right)=0$ for $y=1, \cdots, 2 n-2$. Then we have $e_{2 n-1}=U$. Thus we have

$$
g\left(\left(\nabla_{e_{i}} A\right) e_{j}, U\right)=\beta g\left(e_{j}, P A e_{2}\right)+g\left(e_{j}, P e_{\imath}\right)=g\left(e_{j}, P e_{\imath}\right) .
$$

From this and the equation of Codazzi we find $g\left(P e_{\imath}, e_{\jmath}\right)=0$ and hence

$$
\sum_{i, j-1}^{2 n-2} g\left(P e_{1}, e_{j}\right) g\left(e_{1}, P e_{2}\right)=2 n-2=0 .
$$

This is a contradiction to the fact that $n \geqq 3$.

Next we assume that $u\left(e_{2 n-1}\right)=0$. We then have

$$
g\left(\left(\nabla_{e_{i}} A\right) U, e_{j}\right)=g\left(\left(\nabla_{e_{i}} A\right) e_{3}, U\right)=g\left(e_{\jmath}, P e_{\imath}\right) .
$$

From this and the equation of Codazzi, we have $g\left(P e_{2}, e_{j}\right)=0$. This is also a contradiction. Consequently, we see that rank $L>1$ at some point of $M$.

From (9.14) we see that at most two $\beta_{i}$ can be distinct at each point of $M$. Let us denote them by $\lambda$ and $\mu$. We denote by $p$ the multiplicity of $\lambda$. Then the multiplicity of $\mu$ is $2 n-1-p$.

LEMMA 9.5. Let $H=\lambda_{1}+\alpha$. If $\lambda$ and $\mu$ are constant, $\lambda \neq \mu$, and if $p \geq 2$, $2 n-1-p \geqq 2$, then $\lambda \mu>0$ or $h_{1}=0$. 
Proof. Let $\left\{e_{a}\right\}$ be orthonormal vector fields such that $A e_{a}=\lambda e_{a}+u\left(e_{a}\right) \xi$, $\left\{e_{r}\right\}$ orthonormal vector fields such that $A e_{r}=\mu e_{r}+u\left(e_{r}\right) \xi$ and $\left\{e_{a}, e_{r}\right\}$ a local field of orthonormal frames for $L$. The indices $a, b, c$ and $r, s, t$ run the ranges $\{1,2, \cdots, p\}$ and $\{p+1, \cdots, 2 n-1\}$ respectively.

First of all, we have

$$
\left(\nabla_{e_{a}} A\right) e_{b}+A \nabla_{e_{a}} e_{b}=\lambda \nabla_{e_{a}} e_{b}+g\left(P A e_{a}, e_{b}\right) \xi+u\left(\nabla_{e_{a}} e_{b}\right) \xi+u\left(e_{b}\right) P e_{a},
$$

from which

$$
\begin{aligned}
g\left(\left(\nabla_{e_{a}} A\right) e_{b}, e_{c}\right) & =-g\left(\nabla_{e_{a}} e_{b}, A e_{c}\right)+\lambda g\left(\nabla_{e_{a}} e_{b, e_{c}}\right)+u\left(e_{b}\right) g\left(P e_{a}, e_{c}\right) \\
& =u\left(e_{c}\right) g\left(e_{b}, P e_{a}\right)+u\left(e_{b}\right) g\left(P e_{a}, e_{c}\right) .
\end{aligned}
$$

Therefore the equation of Codazzi implies

$$
u\left(e_{c}\right) g\left(e_{b}, P e_{a}\right)+u\left(e_{b}\right) g\left(P e_{a}, e_{c}\right)-u\left(e_{c}\right) g\left(e_{a}, P e_{b}\right)-u\left(e_{a}\right) g\left(P e_{b}, e_{c}\right)=0 .
$$

from which

$$
2 u\left(e_{c}\right) g\left(e_{b}, P e_{a}\right)+u\left(e_{b}\right) g\left(P e_{a}, e_{c}\right)-u\left(e_{a}\right) g\left(P e_{b}, e_{c}\right)=0 .
$$

Putting $a=c$ in this equation, we have

$$
u\left(e_{a}\right) g\left(e_{b}, P e_{a}\right)=0 .
$$

Similarly we have

$$
u\left(e_{r}\right) g\left(e_{s}, P e_{r}\right)=0
$$

From (9.17) we obtain

$$
\begin{aligned}
0 & =\sum_{a, b} u\left(e_{a}\right) g\left(P e_{a}, e_{b}\right) g\left(e_{b}, P e_{r}\right) \\
& =\sum_{a} u\left(e_{a}\right)\left\{g\left(P e_{a}, P e_{r}\right)-\sum_{s} g\left(P e_{a}, e_{s}\right) g\left(e_{s}, P e_{r}\right)\right\} \\
& =\sum_{a} u\left(e_{a}\right)\left\{-u\left(e_{a}\right) u\left(e_{r}\right)-\sum_{s} g\left(P e_{a}, e_{s}\right) g\left(e_{s}, P e_{r}\right)\right\} .
\end{aligned}
$$

Since $\sum_{a} u\left(e_{a}\right) g\left(P e_{a}, e_{s}\right)=-\sum_{\tau} u\left(e_{r}\right) g\left(P e_{r}, e_{s}\right)=0$ by (9.18), we have

$$
\sum_{a} u\left(e_{a}\right) u\left(e_{a}\right) u\left(e_{r}\right)=0 \text {. }
$$

This shows that $u\left(e_{a}\right)=0$ or $u\left(e_{\tau}\right)=0$.

Without loss of generality we may assume that $u\left(e_{n}\right)=0$ for all $a$ and hence $u\left(e_{r}\right) \neq 0$ for some $r$. Then we have

$$
\begin{aligned}
g\left(\left(\nabla_{e_{a}} A\right) e_{r}, U\right) & =\nabla_{e_{a}} g\left(A e_{r}, U\right)-g\left(A \nabla_{e_{a}} e_{r}, U\right)-g\left(A e_{r}, P A e_{a}\right) \\
& =\mu g\left(\nabla_{e_{a}} e_{r}, U\right)-g\left(\nabla_{e_{a}} e_{r}, A U\right) \\
g\left(\left(\nabla_{e_{r}} A\right) e_{a}, U\right) & =\lambda g\left(\nabla_{e_{r}} e_{a}, U\right)-g\left(\nabla_{e_{r}} e_{a}, A U\right) \\
& =-\lambda \mu g\left(e_{a}, P e_{r}\right)-g\left(\nabla_{e_{r}} e_{a}, A U\right)
\end{aligned}
$$

Therefore the equation of Codazzi implies 


$$
\mu g\left(\nabla_{e_{a}} e_{r}, U\right)+\lambda \mu g\left(e_{a}, P e_{r}\right)-g\left(\nabla_{e_{a}} e_{r}, A U\right)+g\left(\nabla_{e_{r}} e_{a}, A U\right)=0 .
$$

On the other hand, we have

$$
\begin{aligned}
g\left(\nabla_{e_{a}} e_{r}, A U\right) & =\sum_{s} g\left(\nabla_{e_{a}} e_{r}, e_{s}\right) g\left(e_{s}, A U\right)+g\left(\nabla_{e_{a}} e_{r}, \xi\right) g(\xi, A U) \\
& =\mu \sum_{s} g\left(\nabla_{e_{a}} e_{r}, e_{s}\right) g\left(e_{s}, U\right)+g\left(\nabla_{e_{a}} e_{r}, \xi\right) \\
& =\mu g\left(\nabla_{e_{a}} e_{r}, U\right)-g\left(e_{r}, P e_{a}\right) \\
g\left(\nabla_{e_{r}} e_{a}, A U\right) & =-\mu^{2} g\left(e_{a}, P e_{r}\right)-g\left(e_{a}, P e_{r}\right)
\end{aligned}
$$

Substituting these equations into (9.19), we find

$$
\left(\mu^{2}-\lambda \mu+2\right) g\left(P e_{a}, e_{r}\right)=0 .
$$

If $e_{r}=U$, then $A U=\mu U+\xi$. Then, from the definition of $h_{1}$, we have $h_{1}=0$. If $e_{r} \neq U$, then (9.18) shows that $g\left(e_{s}, P e_{r}\right)=0$ for all $s$. From this we see that $g\left(P e_{a}, e_{r}\right)=-g\left(e_{a}, P e_{r}\right) \neq 0$ for some $a$. Consequently, we obtain $\mu^{2}-\lambda \mu+2=0$. Thus we have $\lambda \mu=\mu^{2}+2>0$. This proves our lemma.

LEMMA 9.6. Let $M$ be a pseudo-Einstem hypersurface of $S^{2 n+1}(n \geq 3)$. Then we have $h_{1}=0$.

Proof. By Lemma 9.1, it suffices to show that $H \neq \lambda_{1}+\alpha$. We assume that $H=\lambda_{1}+\alpha$. Then we have (9.14) and the second fundamental form $A$ is represented by (9.12). From (9.14) we see that at most two $\beta_{i}$ are distinct and so we denote them by $\lambda$ and $\mu$. If $\lambda=\mu$ at any point of $M$, then Lemma 9.2 shows that $M$ is totally contact-umbilical. This contradicts to Proposition 2.2. Therefore $\lambda \neq \mu$ at some point. Then, from (9.14) we have

$$
H=\lambda+\mu, \quad \lambda \mu=a-(2 n-1) .
$$

Let $p$ be the multiplicity of $\lambda$. Then we have $H=p \lambda+(2 n-1-p) \mu$. Combining this with (9.21), we have

$$
(p-1) \lambda+(2 n-2-p) \mu=0 .
$$

Suppose $a>(2 n-1)$. Then the second equation of (9.21) shows that $\lambda$ and $\mu$ have the same sign. Therefore (9.22) implies that $p=1$ and $n=3 / 2$. This is a contradiction.

Let $a<(2 n-1)$. If $\lambda=\mu$ at some point, then we have $(2 n-2) \lambda^{2}=a-(2 n-1)$ $<0$ by (9.13). This is a contradiction. Hence there exist exactly two distinct eigenvalues $\lambda, \mu$ of $L$ at each point of $M$. Thus (9.22) implies that $1<p<2 n-2$. From (9.21) and (9.22) we have

$$
\lambda^{2}=-\frac{(2 n-2-p)(a-2 n+1)}{(p-1)}, \quad \mu^{2}=-\frac{(p-1)(a-2 n+1)}{(2 n-2-p)} .
$$

Therefore $\lambda$ and $\mu$ are constant. Thus Lemma 9.5 implies that $\lambda \mu>0$ or $h_{1}=0$. 
If $\lambda \mu>0$, this contradicts to the fact that $\lambda \mu=a-(2 n-1)<0$.

We now assume that $a=(2 n-1)$. Then $\lambda \mu=0$. This gives rank $L \leqq 1$. This is a contradiction by Lemma 9.4. Consequently, we see that $H \neq \lambda_{1}+\alpha$ and hence $h_{1}=0$ by Lemma 9.1. This proves our assertion.

THEOREM 9.1. Let $M$ be a pseudo-Einstem hypersurface of $S^{2 n+1}(n \geq 3)$. Then $M$ has two constant principal curvatures or four constant principal curcatures.

Proof. Since $h_{1}=0$ by Lemma 9.6, the second fundamental form $A$ is represented by a matrix of the form

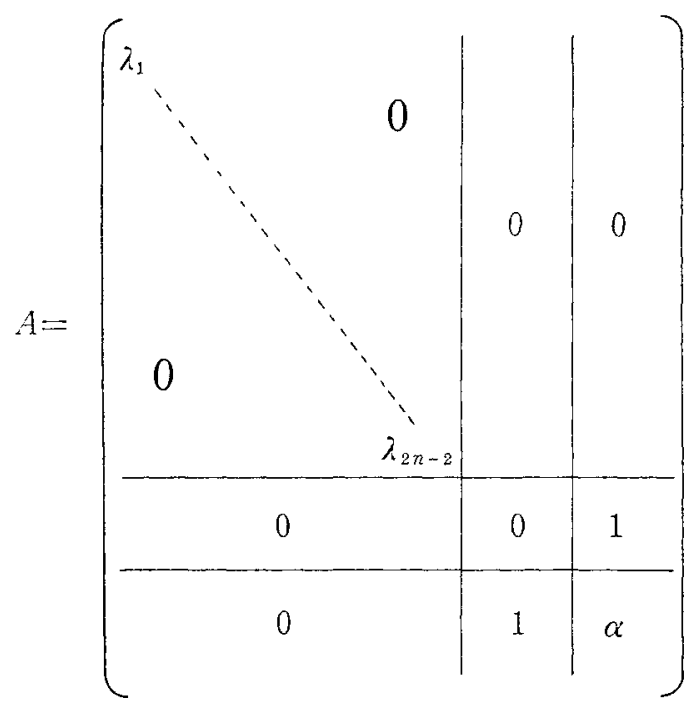

for a local field of orthonormal frames $e_{1}, \cdots, e_{2 n-2}, e_{2 n-1}=\xi, e_{2 n}=U$ of $M$. Then $(9.8),(9.9)$ and $(9.10)$ reduce to

$$
\begin{gathered}
a=(2 n-1)+H \lambda_{i}-\lambda_{\imath}^{2}, \quad \imath=1, \cdots, 2 n-2, \\
a+b=(2 n-2)+H \alpha-\alpha^{2} .
\end{gathered}
$$

On the other hand, from Lemma 9.3, $\alpha$ is a constant. If $\alpha \neq 0$, then $H$ is constant by (9.24). From (9.23) we see that at most two $\lambda_{2}$ are distinct and so we denote them by $\lambda$ and $\mu$. Since $H$ is constant, then $\lambda$ and $\mu$ are both constant by (9.23).

We next assume that $\alpha=0$. Then we have $H=p \lambda+(2 n-2-p) \mu$, where $p$ denotes the multiplicity of $\lambda$.

Suppose $a>(2 n-1)$. If $\lambda \neq \mu$ at some point of $M$, then from $H=\lambda+\mu$, we have $(p-1) \lambda+(2 n-3-p) \mu=0$. Since $\lambda \mu=a-(2 n-1)>0, \lambda$ and $\mu$ have the same sign and hence $p=1, n=4 / 2=2$. This is a contradiction to the fact that $n \geqq 3$. Thus we must have $\lambda=\mu$ at each point. Thus (9.23) implies that $(2 n-3) \lambda^{2}=$ $a-(2 n-1)$, and hence $\lambda$ is a constant. 
Suppose $a<(2 n-1)$. If $\lambda=\mu$ at some point, then we have $(2 n-3) \lambda^{2}=$ $a-(2 n-1)<0$ by $(9.23)$. This a contradiction. Therefore $\lambda \neq \mu$ at each point. Thus we have $H=p \lambda+(2 n-2-p) \mu=\lambda+\mu$ and $\lambda \mu=a-(2 n-1)$. Consequently we have

$$
\lambda^{2}=-\frac{(2 n-3-p)(a-2 n+1)}{(p-1)}, \quad \mu^{2}=-\frac{(p-1)(a-2 n+1)}{(2 n-3-p)},
$$

and hence $\lambda$ and $\mu$ are constant.

We next assume that $a=(2 n-1)$. Then $\lambda \mu=0$. Thus if $\lambda \neq 0$, then $H=p \lambda$ and hence (9.23) implies that $(p-1) \lambda^{2}=0$. Thus we have $p=1$.

We assume that $A e_{2}=0, i=1, \cdots, 2 n-3, A e_{2 n-2}=\lambda e_{2 n-2}$. On the other hand, we have $A U=\alpha U+\xi$. Thus we have

$$
\begin{aligned}
& g\left(\left(\nabla_{e_{i}} A\right) e_{\jmath}, U\right)=-g\left(e_{\jmath}, P e_{\imath}\right), \quad \imath, \jmath=1, \cdots, 2 n-3, \\
& g\left(\left(\nabla_{e_{j}} A\right) e_{\imath}, U\right)=-g\left(e_{\imath}, P e_{\jmath}\right), \quad \imath, \jmath=1, \cdots, 2 n-3 .
\end{aligned}
$$

Hence the equation of Codazzi implies that $g\left(e_{j}, P e_{\imath}\right)=0$. Since $n \geq 3$, we can take $e_{3}$ and $e_{2}$ such that $g\left(e_{3}, P e_{2}\right) \neq 0$. This is a contradiction. Therefore $a \neq(2 n-1)$.

We now consider the matrix $\left(\begin{array}{ll}0 & 1 \\ 1 & \alpha\end{array}\right)$. Then the eigenvalues of this matrix satisfy the quadratic equation

$$
t^{2}-\alpha t-1=0
$$

Let $\lambda=\mu$. Then we have

$$
g\left(\left(\nabla_{e_{i}} A\right) e_{j}, U\right)=-\lambda^{2} g\left(e_{3}, P e_{\imath}\right)+\alpha \lambda g\left(e_{3}, P e_{\imath}\right)+g\left(e_{3}, P e_{\imath}\right) .
$$

From the equation of Codazzi we have

$$
\left(\lambda^{2}-\alpha \lambda-1\right) g\left(e_{y}, P e_{\imath}\right)=0 \text {. }
$$

Therefore we have $\lambda^{2}-\alpha \lambda-1=0$ and hence $\lambda$ satisfies equation (9.25), Thus $M$ has two constant principal curvatures.

Let $\lambda \neq \mu$. We take an orthonormal frame $\left\{e_{a}, e_{r}, U, \xi\right\}$ such that $A e_{a}=\lambda e_{a}$, $A e_{r}=\mu e_{r}$, where $a, b, c=1, \cdots, p ; r, s, t=p+1, \cdots, 2 n-2$. Then we have

$$
\begin{aligned}
& g\left(\left(\nabla_{e_{r}} A\right) e_{a}, U\right)=\alpha \mu g\left(e_{a}, P e_{r}\right)+g\left(e_{a}, P e_{r}\right)-\lambda \mu g\left(e_{a} P e_{r}\right), \\
& g\left(\left(\nabla_{e_{a}} A\right) e_{r}, U\right)=\alpha \lambda g\left(e_{r}, P e_{a}\right)+g\left(e_{r}, P e_{a}\right)-\lambda \mu g\left(e_{r}, P e_{a}\right) .
\end{aligned}
$$

From these equations and the equation of Codazzi we have

$$
(\alpha \lambda+\alpha \mu+2-2 \lambda \mu) g\left(e_{r}, P e_{a}\right)=0 .
$$

If $g\left(e_{r}, P e_{a}\right) \neq 0$ for some $r$ and $a$, then we have

$$
\alpha \lambda+\alpha \mu+2-2 \lambda \mu=0 \text {. }
$$

If $\lambda$ or $\mu$ satisfies (9.25), then we have $\lambda^{2}-\alpha \lambda-1=0$ or $\mu^{2}-\alpha \mu-1=0$. Let $\lambda^{2}-\alpha \lambda-1=0$. Then (9.29) implies that $(\alpha-2 \lambda)(\mu-\lambda)=0$. Since $\lambda \neq \mu$, we have $\lambda=\alpha / 2$. Thus we have $\alpha^{2} / 4-\alpha^{2} / 2-1=0$ and hence $-\alpha^{2} / 4=1$. This is a con- 
tradiction. Consequently $\lambda$ and $\mu$ do not satisfy (9.25). Thus $M$ has four constant principal curvatures.

If $g\left(e_{r}, P e_{a}\right)=0$ for all $r$ and $a$, then have $p \geqq 2$ and $(2 n-2-p) \geqq 2$. In this case, by the similar method used to obtain (9.27), we have $\lambda^{2}-\alpha \lambda-1=0$ and $\mu^{2}-\alpha \mu-1=0$. Therefore $\lambda$ and $\mu$ satisfy (9.25). Moreover, we see that $p$ and $(2 n-2-p)$ are even. Thus the multiplicities of $\lambda$ and $\mu$ are $p+1$ and $2 n-1-p$ respectively and hence they are odd. Consequently, $M$ has two constant principal curvatures or has four constant principal curvatures. This proves our theorem.

\section{$\S 10$. Examples of Pseudo-Einstein hypersurfaces.}

Let $P^{n}(C)$ be a complex projective space of constant holomorphic sectional curvature 4 with almost complex structure $J$. Let $N$ be a $(2 n-1)$-dimensional real hypersurface of $P^{n}(C)$. We denote by $G$ the metric tensor field of $P^{n}(C)$. We denote by the same $G$ the induced metric tensor field of $N$. Let $C^{\prime}$ be a unit normal of $N$ in $P^{n}(C)$. We put $J C^{\prime}=-U^{\prime}$ and $u^{\prime}(X)=G\left(X, U^{\prime}\right)$ for any vector field $X$ tangent to $N$. If the Ricci tensor $S^{\prime}$ of $N$ is of the form $S^{\prime}(X, Y)$ $=\alpha G(X, Y)+\beta u^{\prime}(X) u^{\prime}(Y), \alpha$ and $\beta$ being constant, then $N$ is called a pseudoEinstein real hypersurface of $P^{n}(C)$ (see [4]). We now consider the following commutative diagram:

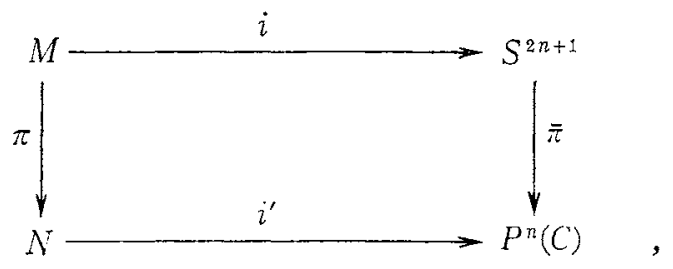

where $M$ is a hypersurface of $S^{2 n+1}$ and $\bar{\pi}, \pi$ denote the Riemannian fibre bundles. We denote by $*$ the horizontal lift with respect to the connection $\eta$. Then, by a straightforward computation, we can show that the Ricci tensor $S$ of $M$ and the Ricci tensor $S^{\prime}$ of $N$ satisfy

$$
\left(S^{\prime}(X, Y)\right)^{*}=S\left(X^{*}, Y^{*}\right)+2 g\left(X^{*}, Y^{*}\right)-2 u\left(X^{*}\right) u\left(Y^{*}\right)
$$

for any vector fields $X$ and $Y$ tangent to $N$. From (10.1) we have the following lemma.

Lemma 10.1. $M$ is a pseudo-Einstern hypersurface of $S^{2 n+1}$ if and only if $N$ is a pseudo-Einstern real hypersurface of $P^{n}(C)$.

Using Lemma 10.1, we give some examples of pseudo-Einstein hypersurfaces of $S^{2 n+1}$.

Let $C^{n+1}$ be the space of $(n+1)$-tuples of complex numbers $\left(z_{1}, \cdots, z_{n+1}\right)$. 
Put $S^{2 n+1}=\left\{\left(z_{1}, \cdots, z_{n+1}\right) \in C^{n+1}: \sum_{j=1}^{n+1}\left|z_{j}\right|^{2}=1\right\}$. For a positive number $r$ we denote by $M_{0}(2 n, r)$ a hypersurface of $S^{2 n+1}$ defined by

$$
\sum_{j=1}^{n}\left|z_{j}\right|^{2}=r\left|z_{n+1}\right|^{2}, \quad \sum_{j=1}^{n+1}\left|z_{\jmath}\right|^{2}=1
$$

For an integer $m(2 \leqq m \leqq n-1)$ and a positive number $s$ a hypersurface $M(2 n, m, s)$ of $S^{2 n+1}$ is defined by

$$
\sum_{j=1}^{m}\left|z_{\jmath}\right|^{2}=s \sum_{j=m+1}^{n+1}\left|z_{j}\right|^{2}, \quad \sum_{j=1}^{n+1}\left|z_{\jmath}\right|^{2}=1
$$

For a number $t(0<t<1)$ we denote by $M(2 n, t)$ a hypersurface of $S^{2 n+1}$ defined by

$$
\sum_{j=1}^{n+1}\left|z_{j}^{2}\right|^{2}=t, \quad \sum_{j=1}^{n+1}\left|z_{j}\right|^{2}=1
$$

$M_{0}(2 n, r)$ and $M(2 n, m, s)$ have two constant principal curvatures and $M(2 n, t)$ has four constant principal curvatures (see [5], [8]).

From the results in [4] and Lemma 10.1 we can see that $M_{0}(2 n, r)$ is always a pseudo-Einstein hypersurface of $S^{2 n+1}$ for any $r$ and $M(2 n, m, s)$ is pseudoEinstein if $s=(m-1) /(n-m)$. Then the Ricci tensor $S$ of $M(2 n, m,(m-1) /(n-m))$ is given by

$$
\begin{aligned}
S(X, Y)= & (2 n-2)[g(X, Y)-\eta(X) \eta(Y)]+\eta(X) S(\xi, Y) \\
& +\eta(Y) S(\xi, X)-\eta(X) \eta(Y) S(\xi, \xi),
\end{aligned}
$$

that is, $a=2 n-2$ and $b=0$. Furthermore $M(2 n, t)$ is pseudo-Einstein if $t=1 /(n-1)$ and the Ricci tensor $S$ of $M(2 n, 1 /(n-1))$ is given by

$$
\begin{aligned}
S(X, Y)= & (2 n-2)[g(X, Y)-\eta(X) \eta(Y)]+(4-4 n) u(X) u(Y) \\
& +\eta(X) S(\xi, Y)+\eta(Y) S(\xi, X)-\eta(X) \eta(Y) S(\xi, \xi),
\end{aligned}
$$

that is, $a=2 n-2$ and $b=4-4 n$.

Moreover $M(2 n, 1 /(n-1))$ is not minimal and $M_{0}(2 n, 2 n-1), M(2 n,(n+1) / 2,1)$ are minimal in $S^{2 n+1}$.

From these considerations we have the following

THEOREM 10.1. If $M$ is a complete pseudo-Einstein hypersurface in $S^{2 n+1}$ $(n \geqq 3)$, then $M$ is congruent to some $M_{0}(2 n, r)$ or to some $M(2 n, m,(m-1) /(n-m))$ ) or to $M(2 n, 1 /(n-1))$.

Proof. From Theorem 9.1 we see that $M$ has two or four constant principal curvatures. If $M$ has two constant principal curvatures, then $M$ is congruent to $M_{0}(2 n, r)$ or $M(2 n, m, s)$ (cf. [6]). Since $M$ is pseudo-Einstein, $M$ is congruent to $M_{0}(2 n, r)$ or to $M(2 n, m,(m-1) /(n-m))$ by the previous argument. If $M$ has four constant principal curvatures, one of the principal curvatures has multiplicity 
1. Therefore, by a theorem of [8], $M$ is congruent to $M(2 n, t)$. Since $M$ is pseudo-Einstein, $M$ is congruent to $M(2 n, 1 /(n-1))$. Therefore we have the theorem.

THEOREM 10.2. If $M$ is a complete pseudo-Einstem mmmal hypersurface in $S^{2 n+1}(n \geqq 3)$, then $M$ is congruent to $M_{0}(2 n, 2 n-1)$ or to $M(2 n,(n+1) / 2,1)$. In the later case, $n$ is odd.

\section{REFERENCES}

[1] A. FIALKow, Hypersurfaces of a space of constant curvature, Ann. of Math., 39 (1938), 762-785.

[2] T. IKawA, Sasakian immersions whth vanishing $C$-Bochner curvature tensors, TRU Math., 11 (1975), 31-34.

[3] I. IshinARA AND M. KON, Contact totally umbilical submanifolds of a Sasakian space form, Annali di Mat., CXIV (1977), 351-364.

[4] M. Kon, Pseudo-Einstein real hypersurfaces in complex space forms, to appear in J. Differential Geometry.

[5] K. Nomizu, Some results in E. Cartan's theory of isoparametric families of hypersurfaces, Bull. of Amer. Math. Soc., 79 (1973), 1184-1188.

[6] P.J. RyAN, Homogenetty and some curvature condition for hypersurfaces, Tôhoku Math. J., 21 (1969), 363-388.

[7] J. Simons, Minimal varieties in riemannian manifolds, Ann. of Math., 88 (1968), $62-105$.

[8] R. TAKAGI, A class of hypersurfaces with constant principal curvatures in a sphere, J. Differential Geometry, 11 (1976), 225-233.

[9] S. TANNo, Constancy of holomorphic sectional curvature in almost Hermitian manifolds, Kōdai Math. Sem. Rep., 25 (1973), 190-201.

[10] K. Yano, On a structure defined by a tensor field $f$ of type $(1,1)$ satisfying $f^{3}+f=0$, Tensor N.S., 14 (1963), 99-109.

[11] K. YaNo AND S. IsHiHARA, Submanifolds with parallel mean curvature vector, J. Differential Geometry, 6 (1971), 95-118.

[12] K. YaNo AND M. Kon, Anti-invariant submanifolds, Marcel Dekker Inc., New York, (1976),

[13] K. Yano AND M. Kon, Generic submanıfolds, to appear in Annali di Mat.

Tokyo Institute of TechNology

AND

HrROSAKI UNIVERSITY 